\title{
Anatomy of the Auditory Thalamocortical System of the Guinea Pig
}

\author{
H. REDIES, S. BRANDNER, AND O.D. CREUTZFELDT \\ Department of Neurobiology, Max-Planck-Institute of Biophysical Chemistry, D-3400 \\ Göttingen, Federal Republic of Germany
}

\begin{abstract}
We investigated the projection from the medial geniculate body (MG) to the tonotopic fields (the anterior field A, the dorsocaudal field DC, the small field $\mathrm{S}$ ) and to the nontonotopic ventrocaudal belt in the auditory cortex of the guinea pig. The auditory fields were first delimited in electrophysiological experiments with microelectrode mapping techniques. Then, small quantities of horseradish peroxidase (HRP) and/or fluorescent retrograde tracers were injected into the sites of interest, and the thalamus was checked for labeled cells.
\end{abstract}

The anterior field $A$ receives its main thalamic input from the ventral nucleus of the MG (MGv). The projection is topographically organized. Roughly, the caudal part of the MGv innervates the rostral part of field A and vice versa. After injection of tracer into low or medium best-frequency sites in A, we also found a topographic gradient along the isofrequency contours: the dorsal (ventral) part of a cortical isofrequency strip receives afferents from the rostral (caudal) portions of the corresponding thalamic isofrequency band. However, it is not so obvious whether such a gradient exists also in the highfrequency part of the projection. A second, weaker projection to field A originates in a magnocellular nucleus that is situated caudomedially in the MG and was therefore named the caudomedial nucleus.

The dorsocaudal field DC receives input from the same nuclei as the anterior field, but the location of the labeled cells in the MGv is different. This was demonstrated by injection of different tracers into sites with like best frequencies in fields A and DC, respectively. After injection of $\mathrm{HRP}$ into the $1-2-\mathrm{kHz}$ isofrequency strip in field $A$ and injection of Nuclear Yellow (NY) into the $1-2-\mathrm{kHz}$ site in field $\mathrm{DC}$, the labeled cells in the MGv form one continuous array that runs from caudal to rostral over the whole extent of the MGv. The anterior part of this array consists of NY-labeled cells; i.e., it projects to field DC. The caudal part is formed by HRP-labeled cells; i.e., it innervates field A. These findings indicate that there is only one continuous tonotopic map in the MGv. This map is split when projected onto the cortex so that two adjacent tonotopic fields (A and DC) result. The cortical maps are rotated relative to the thalamic map in that rostral portions of the MGv project to caudal parts of the tonotopic cortex and vice versa.

The small field $S$ receives its main thalamic input from a region situated in the rostral half of the MG medial to the ventral nucleus (the rostromedial MG). After injection of tracer into the ventrocaudal belt, labeled cells were found dorsal, lateral, and ventral to the MGv. These cells form a continuous band that surrounds the MGv like a shell. A second population of labeled neurons was found in the caudomedial nucleus of the MG.

Similarities and differences between the auditory thalamocortical systems in the guinea pig and other mammalian species are discussed.

Key words: medial geniculate body, auditory cortex, tonotopy, comparative anatomy 
There are three tonotopic fields in the guinea pig auditory cortex (Fig. 1). In the anterior field A, low best frequencies (BFs) are represented anteriorly and high frequencies posteriorly. In the dorsocaudal field DC, the tonotopic gradient is reversed and partly discontinuous: in the dorsal part of DC, the high BFs are situated rostrally, and the low BFs are located immediately caudal to the high BFs. More ventrally in DC, the tonotopy is continuous, with the medium BFs represented as well. The small tonotopic field $\mathrm{S}$ is situated rostral to field A. Though field S occupies less than $1 \mathrm{~mm}^{2}$ of cortical surface, it contains a representation of the whole frequency range. Low BFs are located posteriorly and high BFs anteriorly (Redies et al., '88; see also Kayser and Legouix, '63; Hellweg et al., '77; Robertson and Irvine, '87).

In addition to the tonotopic areas, there are several nontonotopic auditory regions (Fig. 1). The most prominent of these is the ventrocaudal belt (VCB), which is situated caudal to field $A$ and ventral to field $D C$. The neuronal response characteristics in the VCB are different from the tonotopic areas: responsiveness to pure tones is weak, response latencies are longer, and frequency tuning curves are broader (Redies et al., '88).

The aim of the present paper is to describe the thalamic afferents of the tonotopic fields and of the VCB in the guinea pig. In each experimental animal, a $\mathrm{BF}$ map of the relevant auditory area was obtained by recording multiunit responses to pure tones of varying frequencies and amplitudes. Subsequently, small amounts of retrograde tracer substances were injected into sites with known BF. In some animals, different tracers (HRP, Bisbenzimid, Nuclear Yellow) were injected into different fields or into sites with different BFs in one field. Then the thalamus was investigated for occurrence of labeled cells.

The main questions addressed are:

1. Which subnuclei of the auditory thalamus project to fields A, DC, S, and VCB in the guinea pig?

2. Which aspects of the thalamocortical auditory system of the guinea pig exist in other species as well and which are species specific?

Some of the results have been published in preliminary form (Redies and Creutzfeldt, '87; Redies and Brandner,

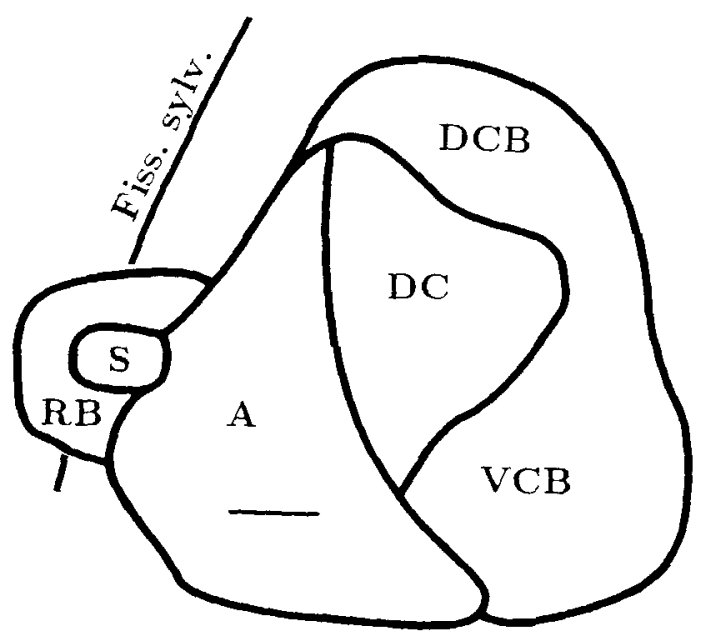

Fig. 1. Schematic representation of the auditory fields in the guinea pig cortex. For orientation, the sylvian fissure is schematically indicated. Calibration bar $1 \mathrm{~mm}$. See our companion paper in this volume for more detailed illustrations.

'87). A detailed account of some of the mapping studies conducted prior to tracer injection is given in Redies et al. ('88).

\section{MATERIALS AND METHODS}

Adult guinea pigs of both sexes with a body weight of 450 $700 \mathrm{~g}$ were used as experimental animals. In each animal, a $\mathrm{BF}$ map of the auditory cortex was obtained prior to tracer injection. Anaesthetics, surgery, and mapping procedures are described in detail in Redies et al. ('88). Therefore, only a short account will be given here.

Initial anaesthesia was achieved with Hypnorm $(1.2 \mathrm{ml}$ ) $\mathrm{kg}$ ) and Valium $(0.4 \mathrm{mg} / \mathrm{kg}$ ) (Green, '75). The saphenous vein and the trachea were cannulated. The animals were then relaxed with Flaxedil and artificially respirated. During the mapping experiment and the survival time, a solu-
Abbreviations

A

AAF

AI

APT

$\mathrm{Bb}$

$\mathrm{BF}$

BF

$\mathrm{DC}$

DCB

EE

EI

FR

HRP

IFS

LGN

LGd

LGv

LP

MG anterior auditory field (guinea pig)

anterior auditory field (cat)

primary auditory field (cat)

area pretectalis

Bisbenzimid

best frequency

cerebral peduncle

dorsocaudal auditory field (guinea pig)

dorsocaudal belt region (guinea pig)

excitatory-excitatory binaural response

excitatory-inhibitory binaural response

fasciculus retroflexus

horseradish peroxidase

isofrequency strip

lateral geniculate nucleus

dorsal nucleus of LGN

ventral nucleus of LGN

lateral posterior nucleus

medial geniculate body ventral nucleus of $\mathrm{MG}$

shell nucleus of MG

medial nucleus of $\mathrm{M}$

rostromedial nucleus of MG (guinea pig)

caudomedial nucleus of MG (guinea pig)

nucleus ruber

Nuclear Yellow

posterior thalamus, lateral part

peripeduncular nucleus

periventricular gray

rostral belt region (guinea pig)

small auditory field (guinea pig)

substantia nigra

substantia nigra, pars compacta

substantia nigra, pars lateralis

substantia nigra, pars reticulata

superior colliculus

ventrocaudal belt region (guinea pig) 
tion containing $0.02 \mathrm{mg} / \mathrm{kg} /$ hour fentanyl base, $8 \mathrm{mg} / \mathrm{kg} /$ hour Flaxedil, and $10 \mathrm{mg} / \mathrm{kg} /$ hour sucrose dissolved in Ringer was administered intravenously.

The skull overlying the temporal cortex was removed and the dura carefully resected. The cortex was covered with warm Agar-Agar (3\% in Ringer) to reduce brain pulsations. $\mathrm{KCl}$-filled glass pipettes $(1-3 \mathrm{M} \Omega$ tested at $1 \mathrm{kHz}$ ) were used as electrodes. Mainly, multiunit activity was recorded. Acoustic stimuli were pure tones of varying frequencies (mostly between 0.5 and $32 \mathrm{kHz}$ ) and amplitudes $(0-80 \mathrm{~dB}$ SPL). The system was calibrated to assure equal stimulus intensity for each frequency. Tuning curves were recorded by an entirely computer-controlled procedure. BFs were determined for 10-60 cortical sites per animal. In two experiments (MSE025, MSE027), a noncalibrated stimulation system was used. Since the maps obtained in these animals are consistent with the other data, they are included here.

\section{Tracer application and survival time}

Horseradish peroxidase (Sigma VI, St. Louis, MO) and the fluorescent substances Bisbenzimid (Riedel de Haen $A G$, Seelze Hannover, West Germany) and Nuclear Yellow (Dr. Loewe, Hoechst AG, Frankfurt-M., West Germany) were used as retrograde tracer substances. In some experiments, only one tracer was applied; in other experiments, two or three different substances were introduced into different cortical sites.

Horseradish peroxidase (HRP) was introduced into the cortex in crystalline form (Mori et al., '80). Elongated glass pipettes with broken tips (inner tip diameter: $20-60 \mu \mathrm{m}$ ) were filled with warm molten paraffin. The paraffin in the pipette became rapidly solid in room temperature. Some of the paraffin was then removed from the pipette's tip by dipping the tip into an ether bath for 5-10 minutes. The hollow space created by this means at the extremity of the pipette was typically $40-80 \mu \mathrm{m}$ deep. It was easily filled with crystalline HRP. Under visual control with the aid of an operation microscope, the pipette was carefully introduced into the cortex in a position chosen by reference to the previously obtained map. It was advanced to a depth of $1-1.5 \mathrm{~mm}$ and left in place for about 2-3 minutes. Then, the pipette was removed and checked under a light microscope to assure that the tracer substance had readily diffused out of its tip. This was always the case.

Bisbenzimid $(\mathrm{Bb})$ was implanted in the same way as HRP. Here, too, after introducing the pipette into the cortex, the substance diffused out of the tip within a few minutes. Nuclear Yellow (NY) is not water soluble and does not diffuse easily when implanted in solid form. Therefore, NY was injected with a $1-\mu$ l Hamilton syringe ( $2 \%$ suspension in distilled water, $0.1-0.2 \mu \mathrm{l}$ ).

After the mapping procedure and tracer application, a survival time of 20-24 hours followed. During this time, animals were kept in the same conditions as in the mapping experiment; i.e., curarizing agents and anaesthetics were administered intravenously, artificial respiration was continued, etc.

\section{Perfusion, histochemistry}

Animals that had only received an injection of HRP, but not of $\mathrm{NY}$ or $\mathrm{Bb}$, were perfused with warm saline, fixative $(1.25 \%$ glutaraldehyde, $1 \%$ paraformaldehyd in phosphate buffer), and a $10 \%$ sucrose solution in phosphate buffer (see Mesulam, '82, Appendix II, p. 125-127, for details). After perfusion, the brain was removed from the skull and immersed in a $20 \%$ sucrose solution for $24-48$ hours. It was then cut on a freezing microtome (frontal sections $40-50 \mu \mathrm{m}$ thick). Further histochemical processing was done according to the TMB procedure of Mesulam et al. ('82, see Appendix III, p. 127-130, for details).

Every second or forth processed slice was coverslipped without further staining, in order to protect the HRP reaction product from deterioration during the alcohol treatment associated with Nissl staining procedures. Another series of adjacent slices was counterstained with thionin (Adams, '80).

When fluorescent substances were used simultaneously, the above protocol was slightly changed: After perfusion with fixative and $10 \%$ sucrose buffer, a perfusion with 300 $400 \mathrm{ml} \mathrm{20 \%}$ sucrose buffer followed. The brain was then removed from the skull and, without further immersion in sucrose solution, directly cut (frontal sections, 40-50 $\mu \mathrm{m}$ thick) on a freezing microtome. Every second or forth slice was immediately mounted and used later (see below) for plotting the cells containing fluorescent substance. By this, we intended to minimize diffusion of $\mathrm{Bb}$ or $\mathrm{NY}$ from the injection site and labeled neurons. The remaining slices were processed for HRP as described above.

\section{Data evaluation}

The caudal thalamus was carefully checked for labeled cells in each animal (light microscope, magnification 250 400). For this, the unstained series were used. Labeled cells were marked on a drawing of the outlines of the slice by means of an X/Y-plotter coupled to the microscope.

The counterstained sections were used to obtain drawings of the cytoarchitectonics of the posterior thalamic region. Accurate localization of labeled cells in relation to cytoarchitectonic borders was done by superimposing drawings of adjacent slices from the unstained and the counterstained series.

\section{RESULTS}

Results are presented from 17 successful experiments. In some cases, only one tracer was injected. In other animals, two or three different substances were used to trace the afferents of several cortical sites, e.g., of locations with corresponding $B F s$ in fields $A$ and $D C$, respectively, or of sites with distinct $B F$ s within one field. Table 1 gives a survey of all experiments.

\section{Subdivisions of the MG}

In order to render the description of our tract-tracing experiments more intelligible, we shall first give a brief survey of the structure of the guinea pig MG. (The reader will find a more comprehensive presentation in the Discussion.) The MG in the guinea pig is an ovoid about $2.7-3 \mathrm{~mm}$ long in the rostrocaudal direction, with a mediolateral diameter of maximally $2.5 \mathrm{~mm}$ and a vertical diameter of $2-2.5 \mathrm{~mm}$. In Figure 2, its cytoarchitecture is illustrated by four frontal sections arranged from rostral (a) to caudal (d). The approximate borders of the different subnuclei are indicated by 
broken lines. The MG of the guinea pig can be subdivided into four nuclei.

The ventral nucleus (MGv, Fig. 2a,b): 'The MGv extends over the rostral two-thirds of the MG. In the rostral part of the MG, it is located laterally. Further caudally, it is slightly displaced medialward by the shell nucleus (see below). The cells are densely packed and not very deeply stained in thionin preparations.

The rostromedial nucleus (MGrm, Fig. 2a): The MGrm is situated in the medial half of the rostral MG. It merges rostrally with the caudal portion of the ventrobasal complex, and distinct borders between these two structures were not recognized. The MGrm can be distinguished from the MGv by the less densely packed and deeper-stained cells.

The caudomedial nucleus (MGcm, Fig. 2b-d): The MGcm can be easily distinguished from the surrounding nuclei by its large and deeply staining cells. It is located medially in the caudal half of the MG, posterior to the rostromedial nucleus. Caudally, the MGcm becomes more and more voluminous and finally occupies nearly the entire caudal pole of the $\mathrm{MG}$.

The above-described three subnuclei can be recognized in Nissl preparations without much ambiguity. However, the anatomical experiments that will be described in the following sections reveal that there is a further subnucleus, which may not be immediately apparent in Nissl material. It surrounds the MGv like a continuous shell from dorsal, lateral, and ventral, and we therefore named it the shell nucleus (MGs, Fig. 2a,b; see Patterson, '77, for shell nucleus in the rat MG). Topographically, this nucleus roughly corresponds to the dorsal, marginal, and ventrolateral subdivisions of the MG as described in the cat (Morest, '64) and rat (Patterson, '77; Ledoux et al., '85, '87). The borders indicated in Figure 2a,b between MGs and MGv and between MGs and MGrm are mainly based on the results of the tract-tracing experiments; in Figure 2c, we are uncertain whether the lateral parvocellular structure belongs to the $\mathrm{MGv}$ and/or to the MGs. See the section entitled Afferents of the Nontonotopic Auditory Cortex and the Discussion for further details.

There is a certain variability between individuals in the size of the MG and its subnuclei. For example, the caudomedial nucleus of the MG was apparent already at $1,400 \mu \mathrm{m}$ posterior to the rostral tip of the MG in some animals but only at $1,700 \mu \mathrm{m}$ in others. Similarly, the borderlines between subnuclei (e.g., MGrm and MGv) may follow slightly different courses in different animals. The reader should be aware of this variability when comparing the photographs of Figure 2 to the section drawings (Figs. 3, 4, etc.) presented in the following paragraphs.

\section{Afferents of the anterior field}

In nine animals, HRP and/or fluorescent tracers were injected into 12 different sites in field A (see Table 1). In the presentation of the results, we follow the BFs of the injection sites. Some animals with several injections into different parts of the cortex will therefore be mentioned repeatedly in the following paragraphs.

Injections into 0.5-2-kHz sites in field $A$. In experiment MSE042, HRP was injected into two sites, $500 \mu \mathrm{m}$ apart from each other, in the ventral part of the $1-2-\mathrm{kHz}$ isofrequency strip (IFS) in field A. In Figure 3a, the BF map of the auditory cortex of this animal is shown; the loci of the HRP injections are indicated by the two crosses. (NY and $\mathrm{Bb}$ were injected into other cortical sites in this case; these
TABLE 1. Survey of Experiments

\begin{tabular}{|c|c|c|c|}
\hline Animal & Tracer & Injection site & Fig. \\
\hline MSE025 & HRP & A, 4-8 kHz, center & 5 \\
\hline MSE027 & HRP & A, $4-8 \mathrm{kHz}$, center & - \\
\hline MSE030 & HRP & $\mathrm{DC}, 1-2 \mathrm{kHz}$, center & 8 \\
\hline MSE035 & HRP & VCB & - \\
\hline MSE036 & $\mathrm{Bb}$ & A, $16-32 \mathrm{kHz}$ ventral & 7 \\
\hline MSE037 & NY & A, $22-32 \mathrm{kHz}$ center & - \\
\hline \multirow[t]{2}{*}{ MSE038 } & $\mathrm{Bb}$ & A, $1-2 \mathrm{kHz}$ ventral & $\ldots$ \\
\hline & HRP & $\mathrm{S}, 11-32 \mathrm{kHz}$ & 12 \\
\hline \multirow[t]{3}{*}{ MSE039 } & NY & $\mathrm{DC}, 1-2 \mathrm{kHz}$, center & 6 \\
\hline & $\mathrm{Bb}$ & A, $5,6-11 \mathrm{kHz}$ strip & 6 \\
\hline & HRP & A, $1-2 \mathrm{kHz}$ strip & 6 \\
\hline \multirow[t]{3}{*}{ MSE042 } & $\mathrm{Bb}$ & $\mathrm{A} / \mathrm{DC}, 32 \mathrm{kHz}$ center & 3 \\
\hline & NY & $\mathrm{DC}, 1.4-2.8 \mathrm{kHz}$ & 3 \\
\hline & HRP & $\mathrm{A}, 1-2 \mathrm{kHz}$ ventral & 3 \\
\hline \multirow[t]{2}{*}{ MSE044 } & $\mathbf{B b}$ & A, $0.5-2 \mathrm{kHz}$ dorsal & 4 \\
\hline & NY & A, $0.5-2 \mathrm{kHz}$ ventral & 4 \\
\hline MSE045 & NY & VCB & - \\
\hline MSE047 & NY & VCB & - \\
\hline MSE048 & $\mathrm{Bb}$ & $\mathrm{RB}$ & - \\
\hline \multirow[t]{2}{*}{ MSE0 04} & HRP & $\mathrm{S}, 8 \mathrm{kHz}$ & - \\
\hline & $\mathrm{Bb}$ & RB & - \\
\hline \multirow[t]{2}{*}{ MSE050 } & HRP & DC, $0.5-1.4 \mathrm{kHz}$ & - \\
\hline & $\mathrm{Bb}$ & A, $1-2 \mathrm{kHz}$ dorsal & - \\
\hline \multirow[t]{2}{*}{ MSE051 } & $\mathrm{Bb}$ & $\mathrm{DC}, 16 \mathrm{kHz}$ & 10 \\
\hline & NY & DC, $1-2 \mathrm{kHz}$ & 10 \\
\hline \multirow{2}{*}{ MSE053 } & $\mathrm{Bb}$ & $\mathrm{VCB}$ & 13 \\
\hline & HRP & VCB & 13 \\
\hline
\end{tabular}

The experiments are listed in chronologic order, with tracer substance(s) used and a short description of the injection site for each animal.

results will be presented in later sections.) Both HRP injection sites had a diameter of about $600-800 \mu \mathrm{m}$ (Fig. 3b). Figure $3 \mathrm{c}$ contains a series of drawings of frontal sections through the posterior thalamus. The HRP-labeled cells are represented by crosses.

HRP labeled cells occurred in two different regions in the caudal thalamus:

1. In the ventral nucleus of the MG, a continuous, densely packed array of labeled neurons was found medially, in the caudal half of the MGv (Fig. 3c, sections 40-46). It begins approximately $900 \mu \mathrm{m}$ behind the rostral pole of the $\mathrm{MG}$ and extends for about $1200 \mu \mathrm{m}$ in the caudal direction.

2. A second population of HRP cells was observed in the caudomedial nucleus of the MG (MGcm). The cells were by far less numerous and much less densely packed than in the MGv (Fig. 3c, section 49). Most of the labeled cells occurred caudally in the MGcm.

Similar results were obtained in animal MSE038: Bb was injected into a $1-2-\mathrm{kHz}$ location in field $\mathrm{A}$, but slightly more ventral than in MSE042. The labeled cells in the MGv form an array that begins about $1,400 \mu \mathrm{m}$ behind the rostral pole of the MG and continues for ca. $600 \mu \mathrm{m}$ caudalward; i.e., the array has a similar position to that it had in the former case but does not extend as far rostrally. Cells in the $\mathrm{MGcm}$ were not evaluated in this case.

In experiment MSE044, a small amount of $\mathrm{Bb}$ was injected dorsally and $1.5 \mu \mathrm{l}$ NY was injected ventrally into the $0.5-2-\mathrm{kHz}$ IFS in field A (Fig. 4a). The Bb injection site had a diameter of ca. $500 \mu \mathrm{m}$ and the NY injection one of

Fig. 2. The cytoarchitecture of the guinea pig MG is shown in four frontal sections (50 $\mu \mathrm{m}$ thick, counterstained with thionin). The distance of the sections relative to the rostral tip of the MG is (a) $600 \mu \mathrm{m}$, (b) $1,500 \mu \mathrm{m}$, (c) $2,300 \mu \mathrm{m}$, and (d) $2,700 \mu \mathrm{m}$. Calibration bar in a is 0.5 $\mathrm{mm}$. 

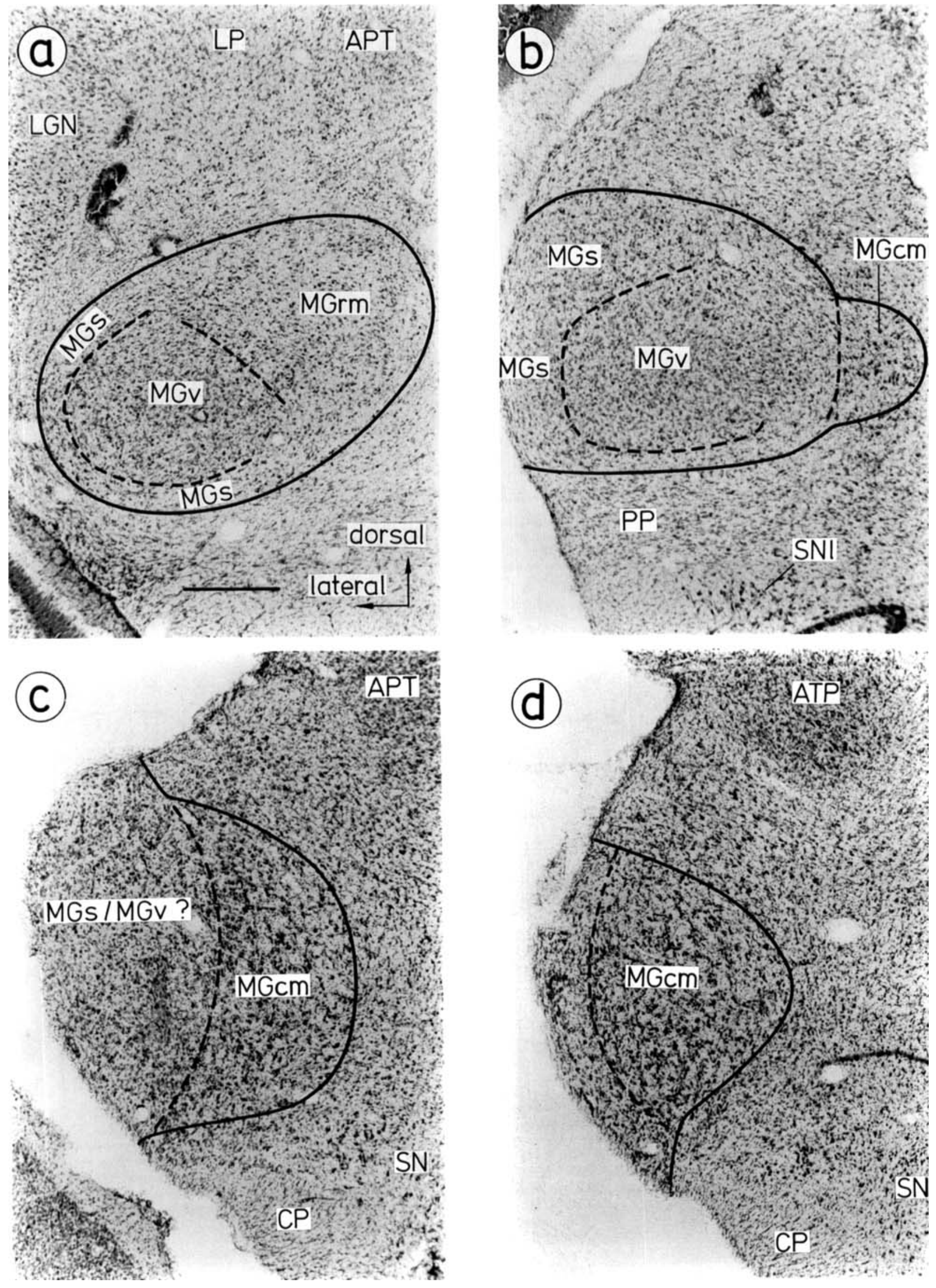

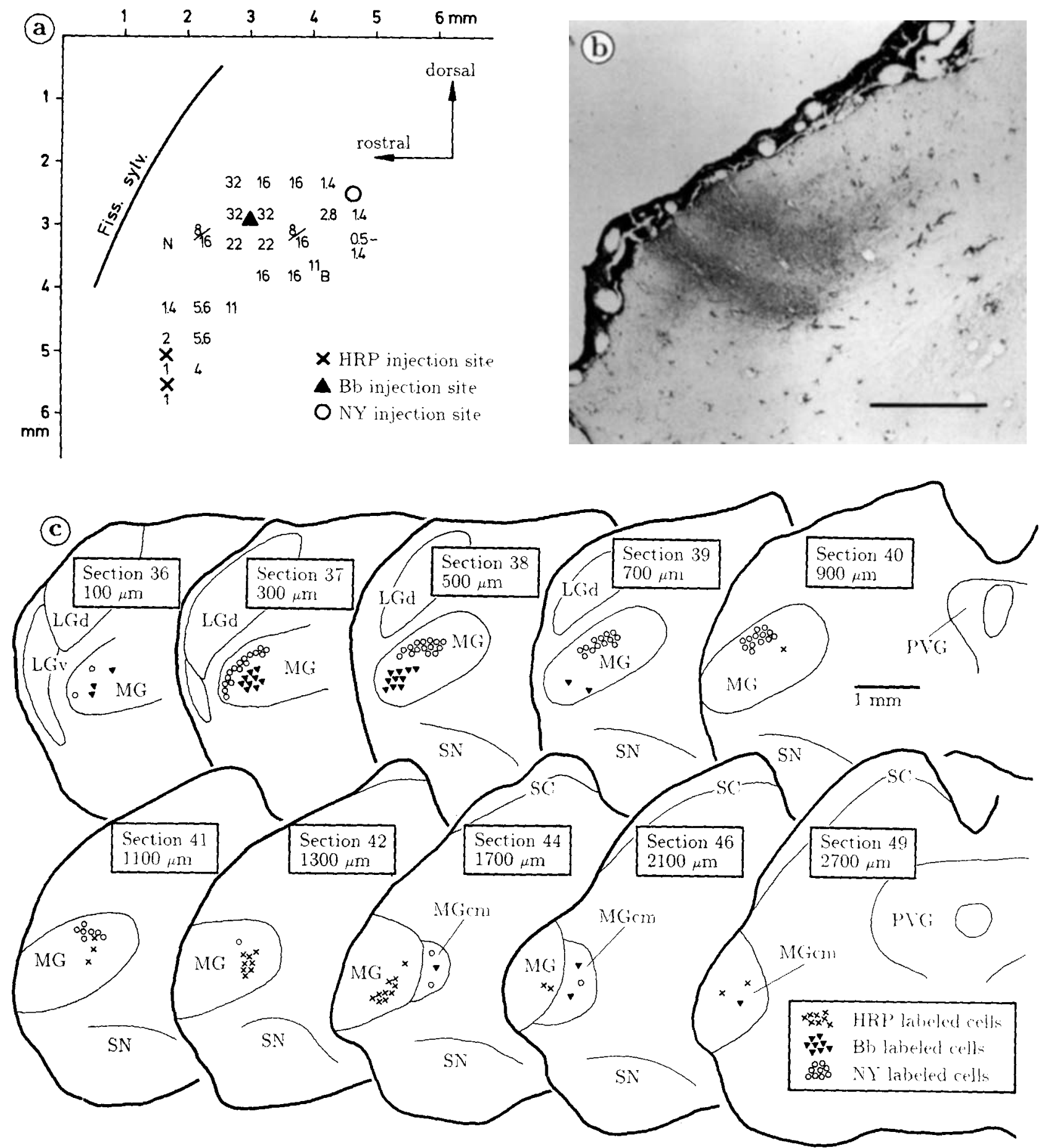

Fig. 3. Experiment MSE042. a: The BF map of animal MSE042 (a surface view of the temporal cortex). For orientation, the sylvian fissure is schematically indicated. Each recording site is represented by a symbol. Numbers mean BFs in kHz; numbers separated by a slash (e.g., 8/ 16) stand for double-peaked tuning curves with two "BFs." $B$ means broad tuning curves and $N$ no response to pure tones. HRP was injected into two sites ventrally in the $1-2-\mathrm{kHz}$ IFS (crosses) in field $\mathrm{A}$; $\mathrm{Bb}$ was injected into the borderline between fields $\mathrm{A}$ and DC (filled triangle); and NY was injected into a 1.4-2.8-kHz site in field DC (empty circle). b: Photomicrograph of the center of the HRP injection sites. The section is not counterstained. Calibration bar $1 \mathrm{~mm}$. c: Frontal sections through the posterior thalamus. The sections are ordered from rostral (upper left) to caudal (lower right). The section number and the distance relative to the rostral pole of the $\mathrm{MG}$ are indicated on each section. Each symbol represents three to ten labeled neurons. 


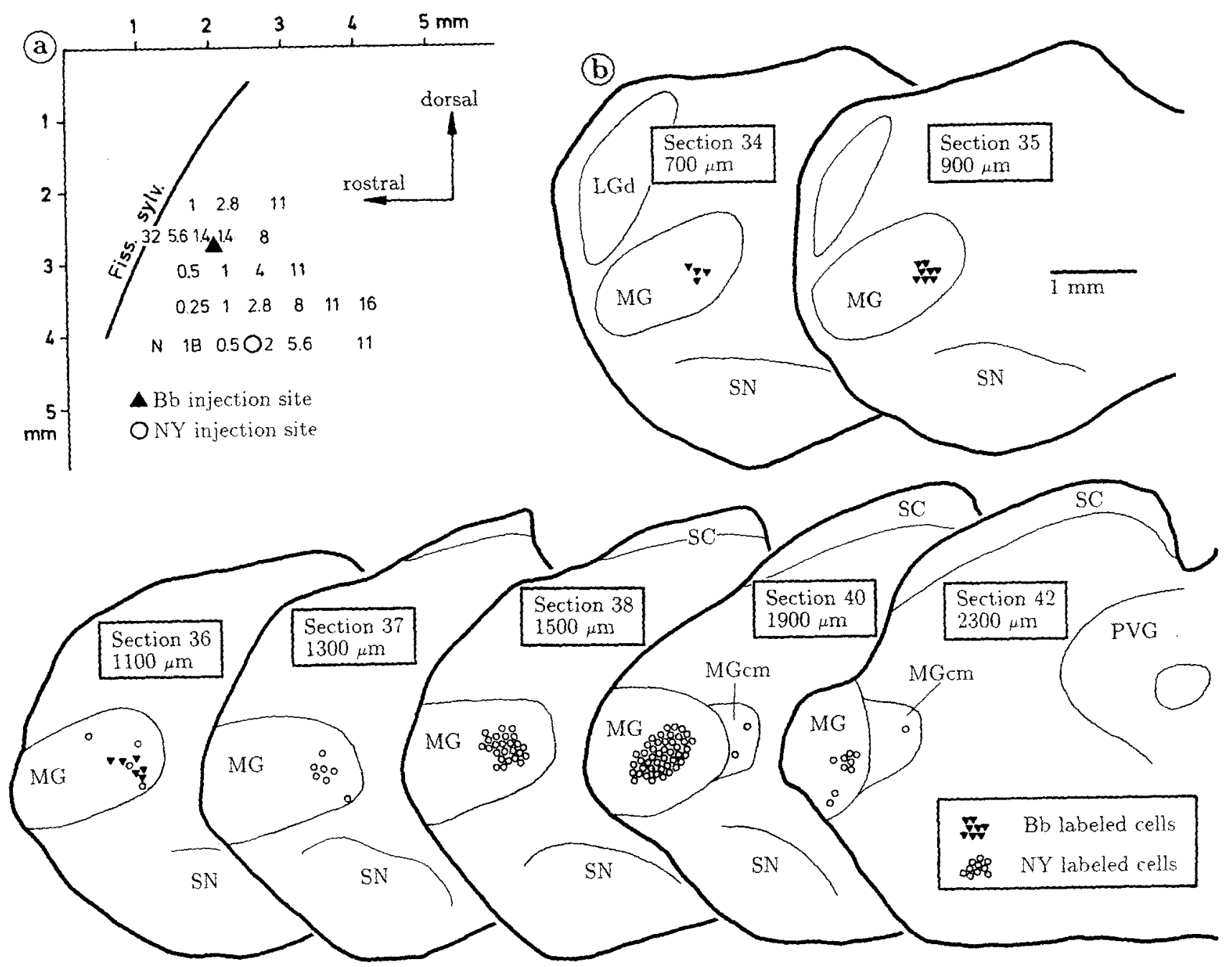

Fig. 4. Experiment MSE044. a: The BF map of animal MSE044. Mainly, field A was mapped in this experiment. NY was injected ventrally and $\mathrm{Bb}$ dorsally in to the $0.5-2-\mathrm{kHz}$ IFS in field A. b: Frontal sec-

about 1-1.5 mm. The plots of the NY-and Bb-labeled cells in the MGv are shown in Figure $4 b$. The NY cells are more numerous than the $\mathrm{Bb}$ cells, probably due to the larger diameter of the NY injection site. A clear spatial separation of the $\mathrm{Bb}$ and NY cells is apparent: the labeled thalamic band consists of $\mathrm{Bb}$ cells rostrally and of NY cells caudally. This means that the caudal part of the thalamic $0.5-2-\mathrm{kHz}$ band projects to the ventral $0.5-2-\mathrm{kHz}$ IFS in $\mathrm{A}$, while the more rostral parts of the thalamic band innervate more dorsal parts of the cortical IFS. The labeled cells in the MGcm in MSE044 are loosely scattered over the whole rostrocaudal extent of this nucleus, but most of the labeled cells are again concentrated in the caudal part of the MGcm, about 2,500$2,900 \mu \mathrm{m}$ behind the rostral pole of the MG. (The caudal part of the MGcm is not shown in Fig. 4b.) A comparison of the respective locations of the $\mathrm{Bb}$ and $\mathrm{NY}$ cells is not possible, since we found only two labeled $\mathrm{Bb}$ cells in the $\mathrm{MG} \mathrm{cm}$. tions through the posterior thalamus. Each symbol (triangles and circles) stands for five to ten labeled cells. Other explanations as in Figure 3.

Injections into $4-11-k \mathrm{~Hz}$ sites in field $A$. In three animals, the afferents to the medium IFS in field $A$ were investigated. As a representative case, MSE025 will be described first. HRP was injected into a $4-8-\mathrm{kHz}$ location in the center of field A (Fig. 5a). The HRP-labeled cells in the MGv form a continuous, densely packed column that begins about $500-600 \mu \mathrm{m}$ behind the rostral pole of $\mathrm{MG}$ and extends for ca. $500 \mu \mathrm{m}$ caudally (Fig. 5b, sections 44-50). Figure 5c,d shows photomicrographs of the labeled cells from section 46 in Figure $5 \mathrm{~b}$. Some HRP-filled cells were again observed in the $\mathrm{MGcm}$ (Fig. $5 \mathrm{~b}$, sections 56, 58). In MSE027, HRP was injected in a location very similar to the former case. As could be expected, results were very much alike.

In experiment MSE039, four injections of $\mathrm{Bb}$ were done into the center and the ventral portion of the $5.6-11-\mathrm{kHz}$ strip of field $A$, in intervals of $0.5 \mathrm{~mm}$ (Fig. 6a, triangles). 

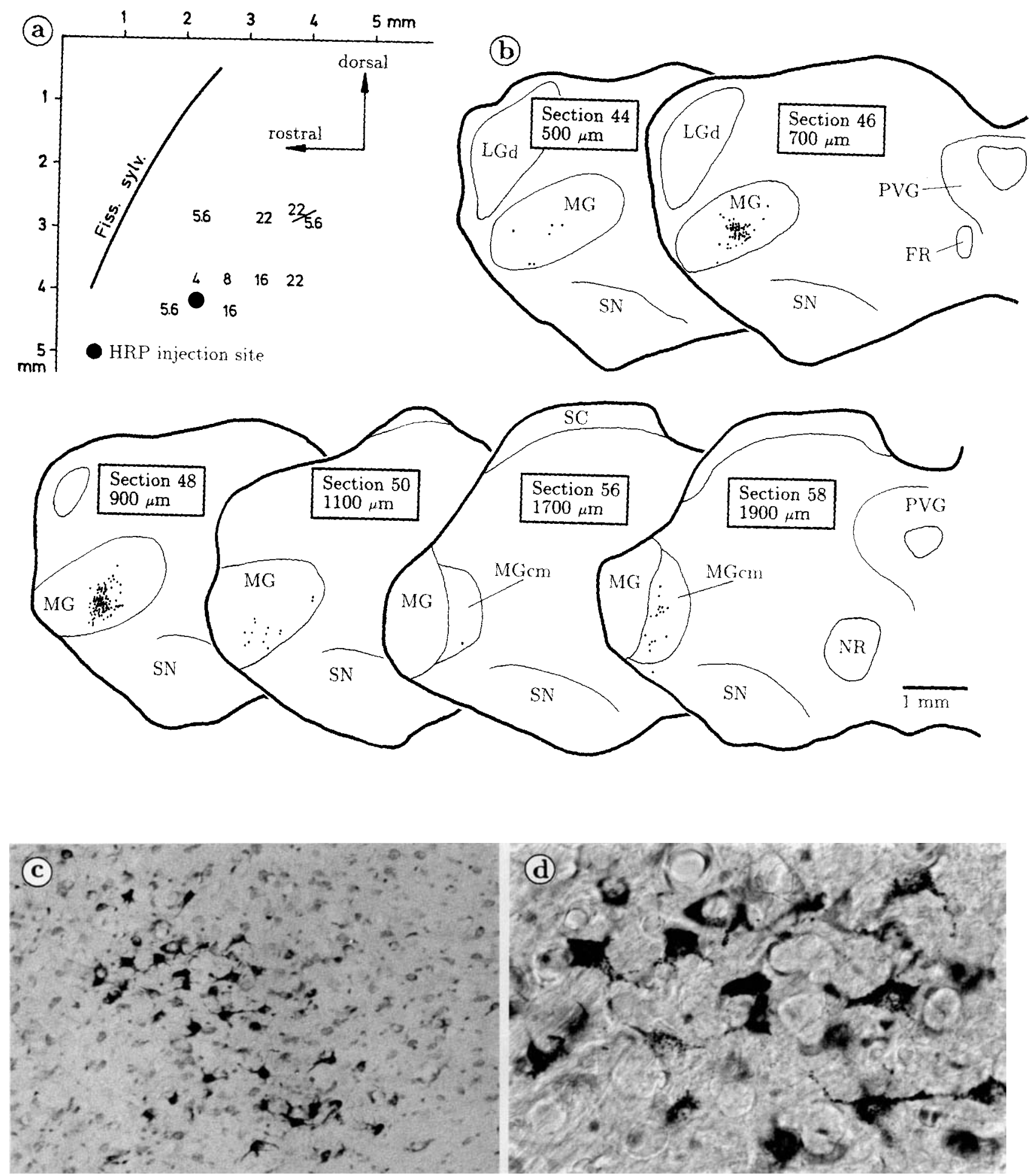

Fig. 5. Experiment MSE025. a: The BF map of animal MSE025. HRP was injected into the center of field $A$ in this experiment. b: Frontal sections through the posterior thalamus. Each HRP-labeled cell is

represented by a dot. c,d: Photomicrographs of section 46 (c) magnification 125:1, (d) magnification 490:1. Other explanations as in Figure 3. 

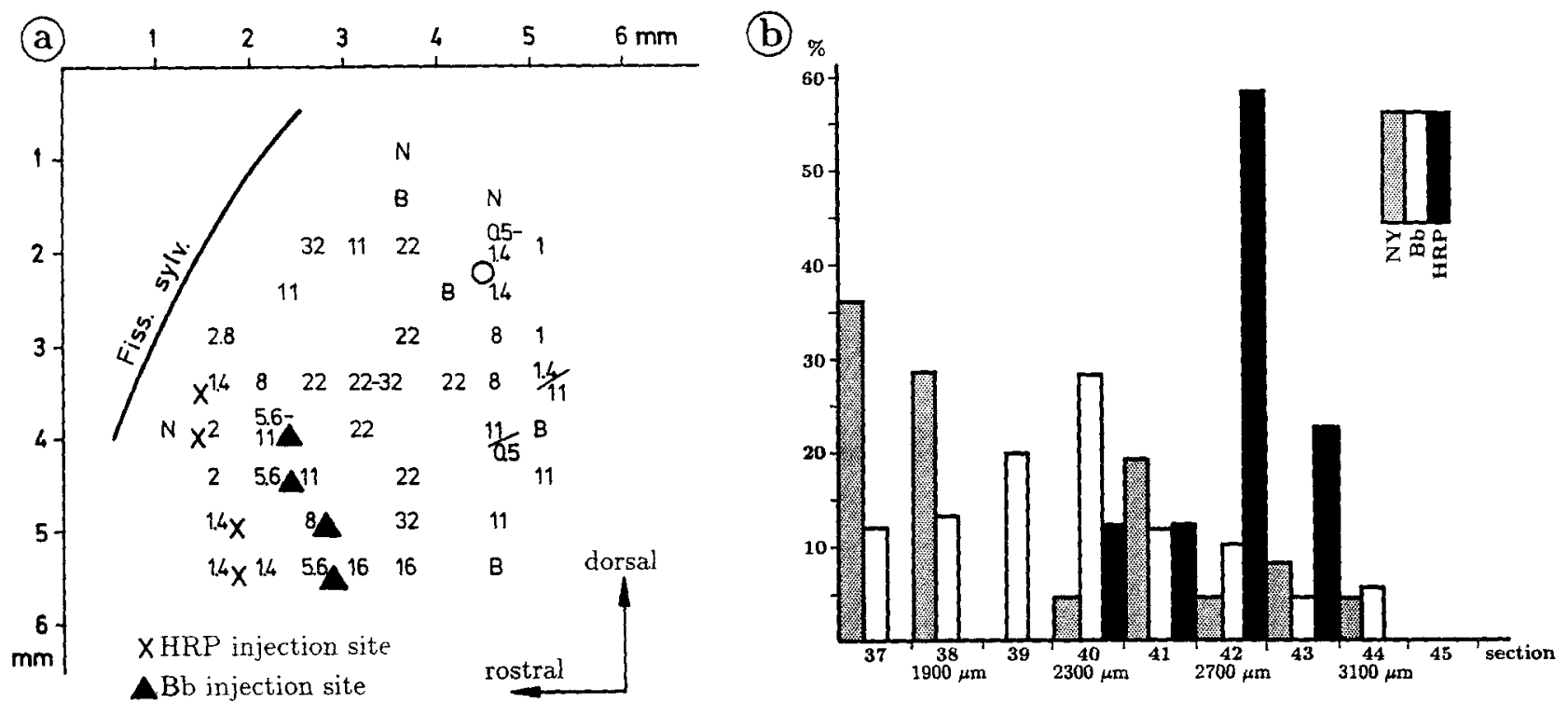

ONY injection site

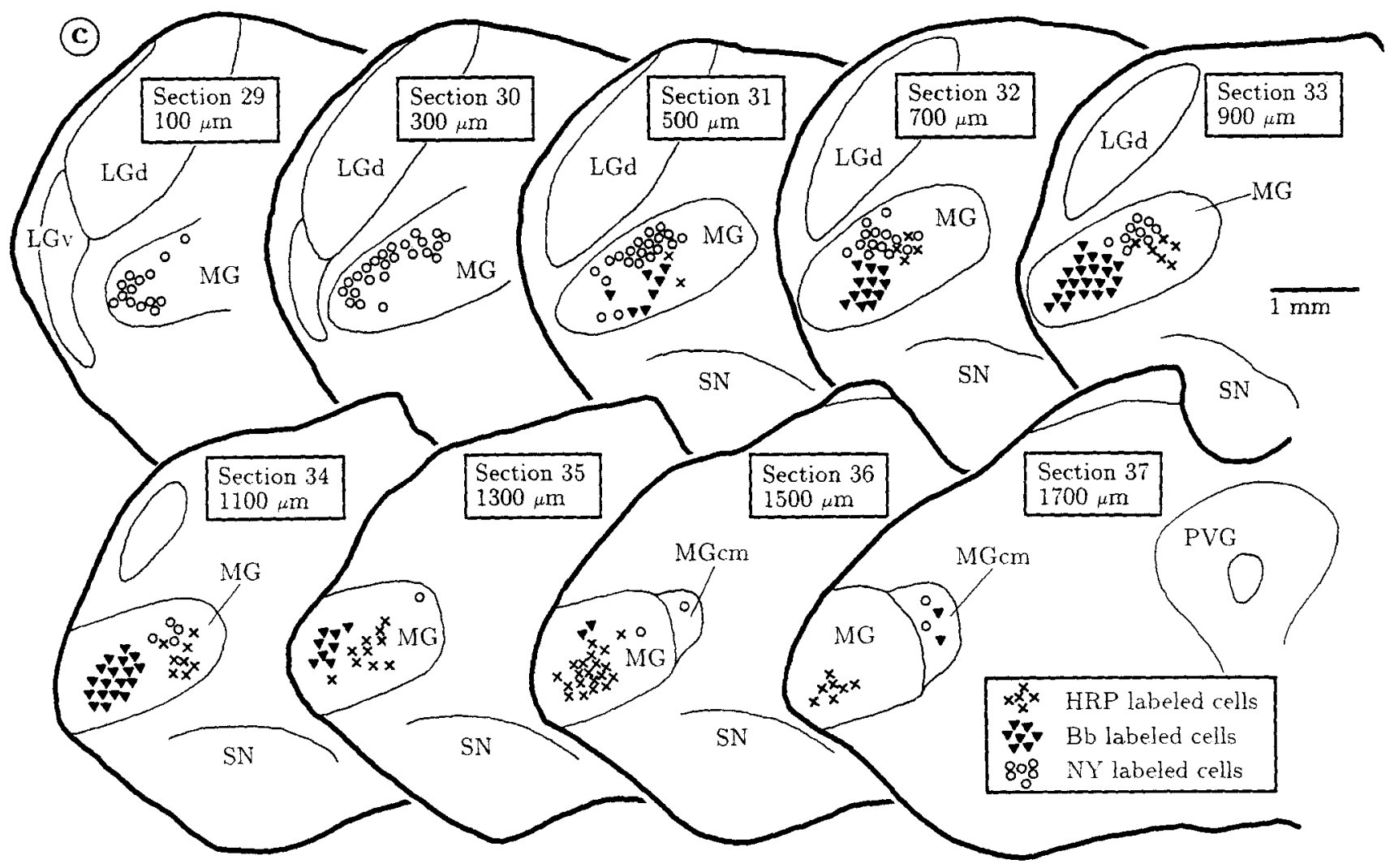

Fig. 6. Experiment MSE039. a: The BF map of the auditory cortex. HRP was injected into four low-frequency sites and $\mathrm{Bb}$ into four medium BF sites in field A. NY was injected into a low-frequency location in field DC. b: The histogram represents the spatial distribution of the differently labeled cells found in the $\mathrm{MGcm}$. Each column gives the percentage of the cells labeled by a specific tracer in a given section relative to all cells labeled by this tracer in the $\mathrm{MGcm}$. There were $28 \mathrm{NY}$ cells, $66 \mathrm{Bb}$ cells, and $28 \mathrm{HRP}$ cells in total. Below the abscisss, the section numbers and the distance of the sections relative to the rostral pole of the MG are indicated. c: Frontal sections through the posterior thalamus. Each symbol stands for three to ten labeled neurons (see inset). Only the rostral end of the MGcm is shown (section 36/37). Other explanations as in Figure 3. 

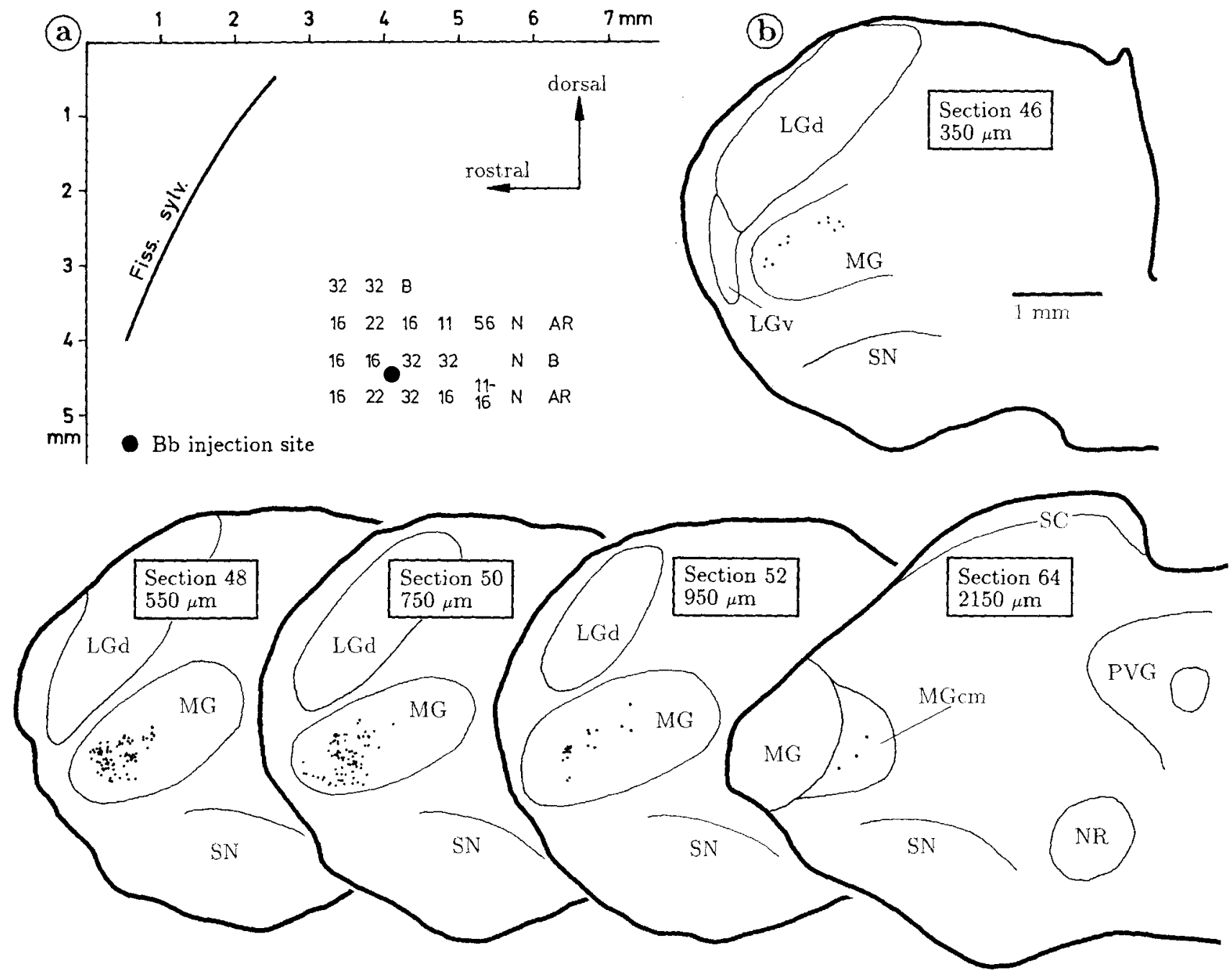

Fig. 7. Experiment MSE036. a: The BF map of animal MSE036. Bb was injected into a $16-32-\mathrm{kHz}$ location ventrally in field $\mathrm{A}$. b: Frontal sections through the posterior thalamus. Each Bb-labeled neuron is represented by a dot. See Figure 3 for further explanations.
The $\mathrm{Bb}$-labeled cells in the MG are represented by triangles in Figure 6c. Rostrally, the labeled array has a similar position to that of the HRP cells in MSE025 and MSE027. It then continues caudolaterally.

Thus, the labeled cells in the MGv observed after medium IFS injections lie rostrolateral to the cells found after injections into low IFS. A comparison of cases MSE025, MSE027, and MSE039 shows that the topography of the 4$11-\mathrm{kHz}$ projection is similar to the $0.5-2-\mathrm{kHz}$ projection: The rostromedial part of the $4-11-\mathrm{kHz}$ thalamic array is connected to the dorsal portion and the caudolateral part to the ventral portion of the corresponding IFS in field A.

Injections into high-frequency sites in field $A$. In MSE036, $\mathrm{Bb}$ was injected into a $16-32-\mathrm{kHz}$ site ventrally in field A (Fig. 7a). The main bulk of labeled cells was found rostrally in the MGv. It begins about $350 \mu \mathrm{m}$ posterior to the rostral tip of MGv (Fig. 7b, section 46) and continues caudally for ca. $600 \mu \mathrm{m}$. The cell mass has a spherical shape; slight discontinuities are apparent in it, particularly in section 48: the cells are grouped into several clusters, which are separated by gaps where fewer or no labeled cells occur. Again, some scattered cells were found in the $\mathrm{MGcm}$, mainly in its rostral half. A similar picture emerges from experiment MSE037, where NY was injected into the center of the $22-32-\mathrm{kHz}$ IFS. Also here, a tendency of the labeled cells to form distinct clusters was noted.

An injection of $\mathrm{Bb}$ into the center of the $32-\mathrm{kHz}$ strip, i.e., into the borderline between fields $A$ and $D C$, was done in experiment MSE042 (Fig. 3). The diameter of the injection site was about $600-800 \mu \mathrm{m}$. Results were similar to MSE036 and MSE037, but the labeled cells were situated still more rostrally (Fig. 3c, triangles in sections $36-39$ ). We want to emphasize that though $\mathrm{Bb}$ probably diffused into the highfrequency regions of fields $\mathrm{A}$ and $\mathrm{DC}$, there was only one array of labeled cells in the MGv. Discontinuities were not observed in this case.

A topographic gradient along the IFS, as observed after injections into low and medium BF locations, is not so evident: In case MSE036 (Fig. 7), where the ventral 16-32-kHz site was injected, labeled cells occur approximately in the 

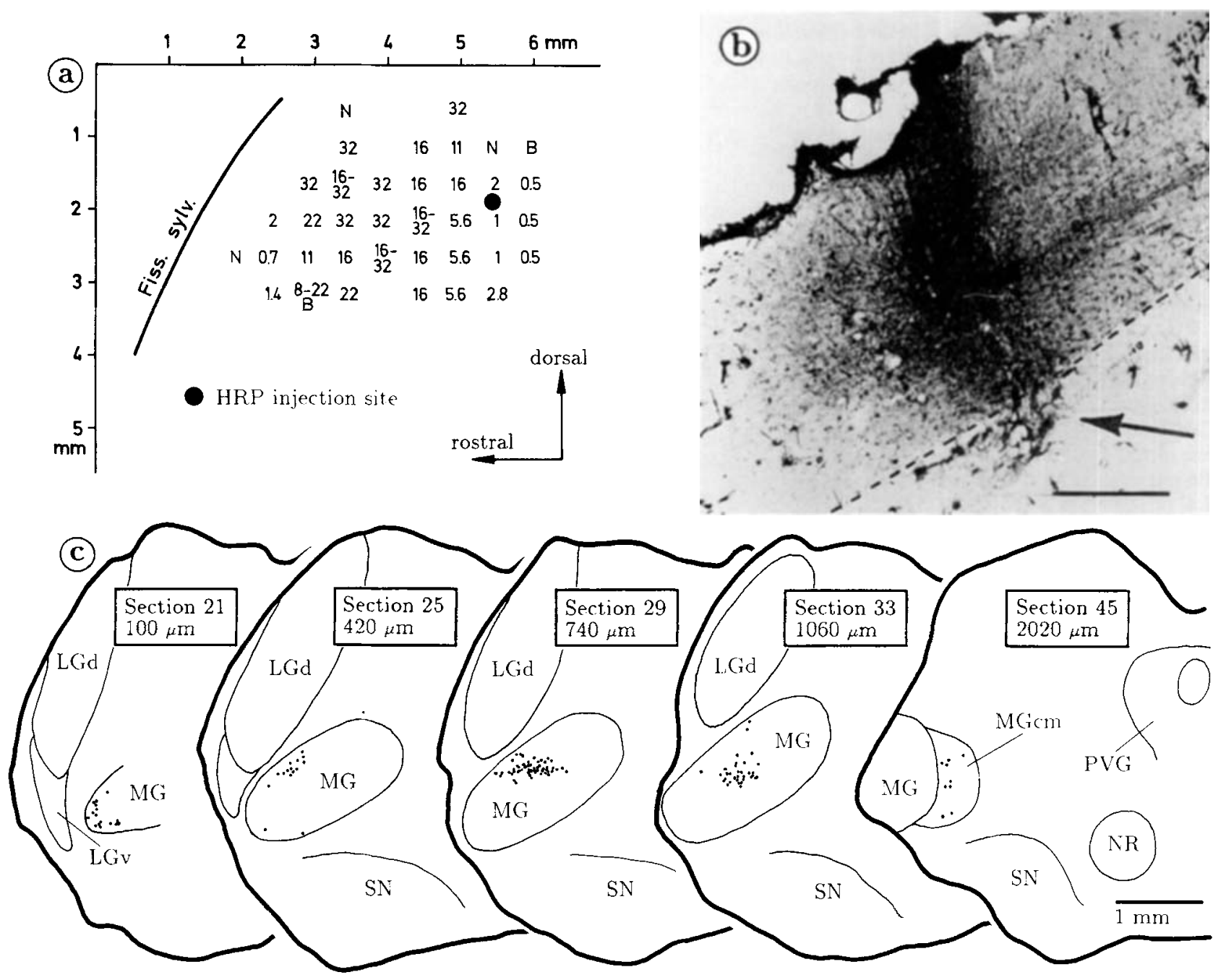

Fig. 8. Experiment MSE030. a: The BF map of MSE030. HRP was injected into a $1-2-\mathrm{kHz}$ location in field DC. b: Photomicrograph of the center of the HRP injection site. The section is not counterstained. For orientation, the transition between layer VI and the white matter is indi- cated by a broken line. The arrow points to labeled fibers that leave cortical layer 6 and enter the white matter. Calibration bar $0.5 \mathrm{~mm}$. c: Frontal sections through the posterior thalamus. Each dot represents one HRP-labeled cell. See Figure 3 for further details.

There is also some order in the projection from the MGcm to field A, though the topography is much less strict: the labeled cells observed after injections into low-frequency regions of field A tend to occur caudally, whereas cells projecting to higher-frequency regions were situated somewhat more rostrally in the $\mathrm{MGcm}$.

\section{Afferents of the dorsocaudal field}

In five animals, $\mathrm{HRP}, \mathrm{Bb}$, and/or $\mathrm{NY}$ were injected into various sites in the dorsocaudal field $\mathrm{DC}$. As in field $\mathrm{A}$, the isofrequency strips in DC are oriented from dorsal to ventral (Redies et al., ' 88 ). Since DC measures only about $2.5 \mathrm{~mm}$ in the dorsoventral direction, we always placed the injections into the center of the IFS in order to avoid diffusion beyond the borders of the field.

As a representative case, animal MSE030 will be described first (Fig. 8). HRP was injected into a $1-2-\mathrm{kHz}$ location in field DC (Fig. 8a). The injection site had a diameter same or a similar principle also holds for the high-frequency part of the projection must be left open at this point. 
of about $1 \mathrm{~mm}$ (Fig. 8b). In Figure 8c, drawings of frontal sections through the posterior thalamus of this animal are reproduced. The HRP-containing cells are represented by dots. Photomicrographs of some of the frontal sections are shown in Figure 9. HRP-containing cells were observed mainly in the ventral and in the caudomedial nucleus of the MG.

The first labeled cells occurred very rostrally in the MGv. In frontal sections, they form a curved array that runs along the outer margin of the nucleus (section 21 in Figs. 8c, 9). The column of labeled neurons then heads caudalward, occupying a dorsomedial position in the MGv (Figs. 8c, 9). It ends about halfway between the rostral and the caudal tip of the MG.

Still further caudally, in the MGcm, another population of labeled cells was found (Fig. 8c, section 45). The HRP. filled cells are less numerous and less densely packed than in the MGv. They are widely scattered over the rostrocaudal extent of the MGcm, but most cells occur in the rostral half of the nucleus. A few scattered cells were also found dorsomedial of the MGv, ca. $1,200-1,500 \mu \mathrm{m}$ caudal of the rostral pole of the MG (not shown in Fig. 8c).

Two other experiments with tracer injection into low BF locations in field DC yielded similar results to those for MSE030. These experiments are of special interest for the topography of the projection from the MGv to fields $\mathrm{A}$ and $D C$ and will be discussed in detail later.

In case MSE051, $\mathrm{Bb}$ was injected into a $16-\mathrm{kHz}$ location and $0.1 \mu \mathrm{l}$ of NY was injected into the low-frequency part of field DC (Fig. 10). This was done in order to see whether the abrupt skip from high to low frequencies in the dorsal part of DC (see the beginning of this paper and maps in Figs. 8a, 10a; see also Redies et al., ' 88 ) has an equivalence in the MGv. This is in fact the case, as shown in Figure 10b. The $\mathrm{Bb}$-labeled cells (triangles) build an array shaped like a horseshoe in the rostral MGv. The NY-labeled neurons (open circles) are located immediately lateral and laterodorsal of the Bb cells. In other words, in the part of the MGv that projects to field DC, the neurons with low BFs lie directly beside the neurons with high BFs. The labeled cells in the MGcm in this animal are widely scattered over a rostrocaudal extent of about $1,000 \mu \mathrm{m}$. We did not see any spatial separation between the NY and the Bb cells; again, most of the labeled cells occur in the rostral half of the $\mathrm{MGcm}$.

Similar results were obtained in case MSE042 (see Fig. $3 a, c)$. Two tracers were injected into regions with high (32 $\mathrm{kHz}, \mathrm{Bb})$ and low $(1.4-2.8 \mathrm{kHz}, \mathrm{NY}) \mathrm{BF}$ 's, respectively. Again, the cell clusters in the MGv lie in close proximity (Fig. 3c, sections 37,38), but there is, in contrast to case MSE051, a small gap between them. Probably, this gap corresponds to the $16-\mathrm{kHz}$ cell column labeled in case MSE051 (Fig. 10).

In summary, field DC receives its projection from the $\mathrm{MGv}$ and from the $\mathrm{MGcm}$, i.e., from the same nuclei as field A. But the cells projecting to field DC occupy another position in the MGv than those projecting to field $\mathrm{A}$, as will be further analyzed in the following paragraph.

Fig. 9. Photomicrographs of frontal sections through the ventral MG of MSE030 (76-fold magnification; the sections are not counterstained). The numbers in the upper left (S21, S23, etc.) indicate the section from which the photograph was taken. Compare with Figure 8c.
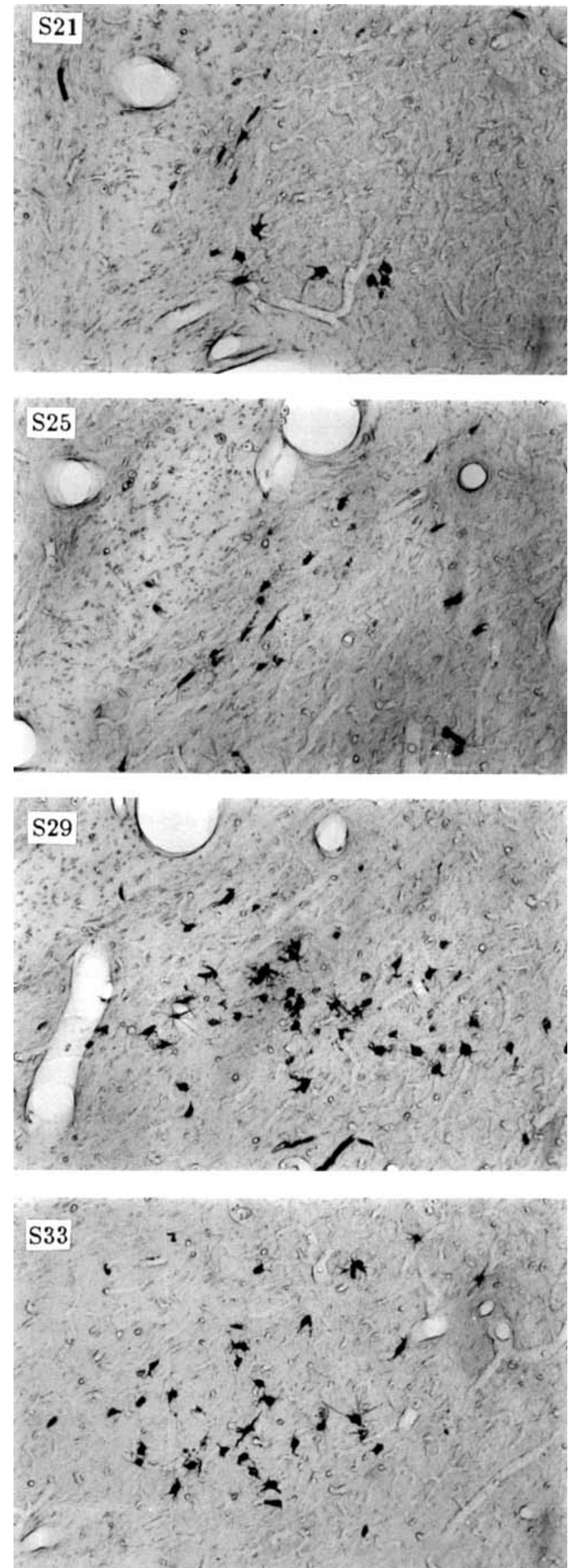

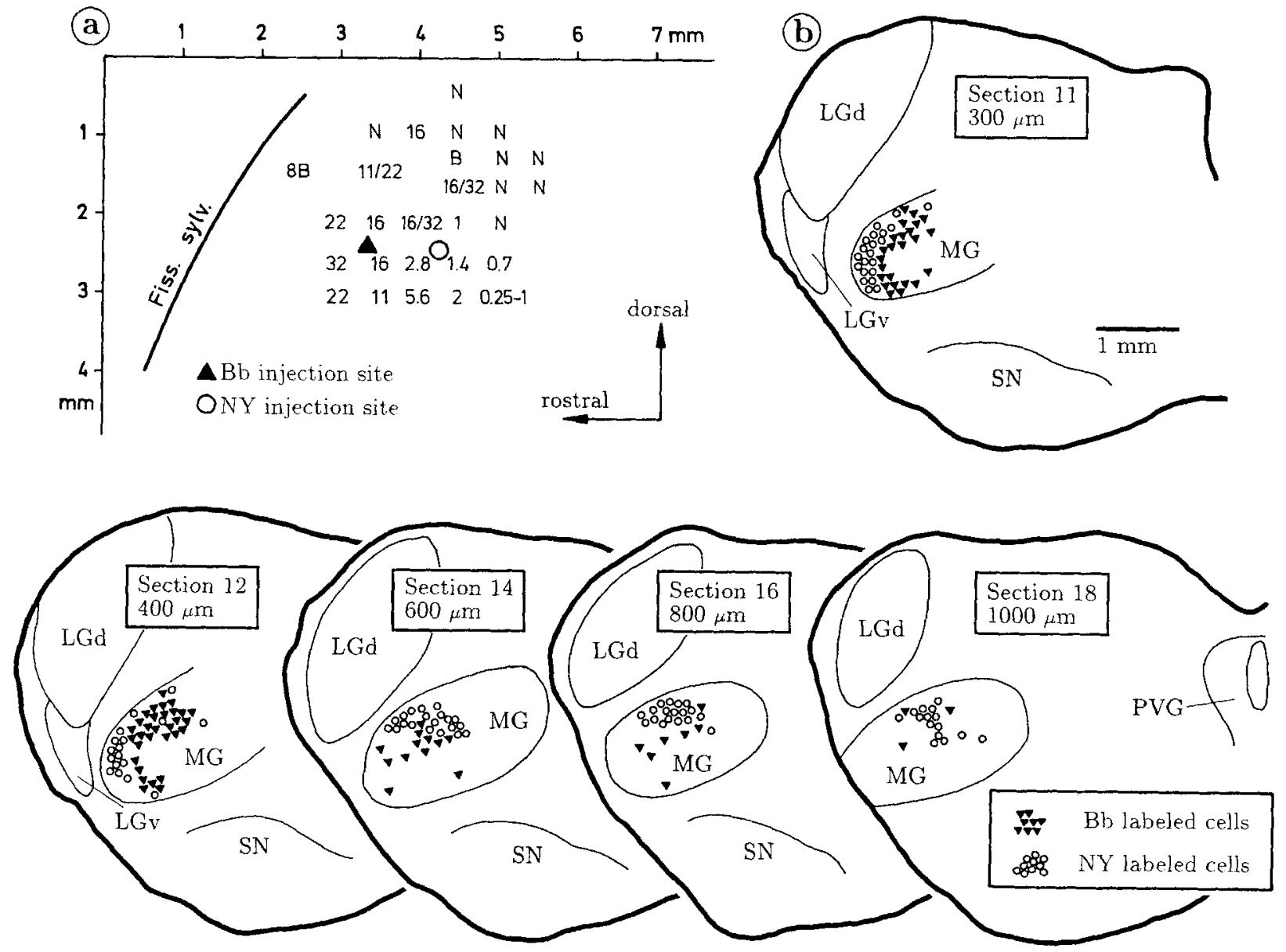

Fig. 10. Experiment MSE051. a: The BF map of MSE051. NY was injected caudally and $\mathrm{Bb}$ rostrally into field $\mathrm{DC}$. b: Frontal sections through the posterior thalamus. Each empty circle stands for three to

\section{Topography of the projection to fields A and DC}

A comparison of the projection from the MGv to fields A and DC in different animals shows that the respective cell groups in the MGv are spatially separated. For example, in case MSE030 (Fig. 8), the main bulk of the MG cells projecting to the low-frequency site in field DC is located in the rostral half of the MGv. In case MSE044 (Fig. 4), where tracer was injected into the low IFS in field $A$, the main mass of the cells occurs in the caudal two-thirds of the MGv.

Experiment MSE039 (Fig. 6) was done in order to demonstrate the projections from the MGv to sites with like BFs in fields $A$ and DC in one animal. Four small HRP injections were done along the $1-2-\mathrm{kHz}$ isofrequency strip in field $\mathrm{A}$. NY was injected in field DC, also in a $1-2-\mathrm{kHz}$ site. As illustrated in Figure 6c, the NY cells projecting to DC form an array very similar to that found in case MSE030 (see Fig. $8 \mathrm{c})$ : a row of labeled cells first follows the lateral contours of MGv and then heads dorsocaudally. The HRP-labeled cells projecting to field A are located caudally in the MGv. Both arrays meet halfway between the rostral and the caudal tip of the MGv (Fig. 6c, sections 32-34). At this level, the Bb ten NY-labeled neurons and each filled triangle for three to ten $\mathrm{Bb}$ cells. Further explanations as in Figure 3.

and NY cells slightly intermingle, but the majority of the cells projecting to field DC are located dorsolaterally from those projecting to field A. Double-labeled cells were not found, with one (dubious) exception.

In experiment MSE050, $\mathrm{Bb}$ was injected dorsally in a 1$2-\mathrm{kHz}$ position in field $\mathrm{A} ; \mathrm{HRP}$ was injected into a $0.5-1.4$ $\mathrm{kHz}$ site in field DC. Results were similar to those in the above-described case, but the array of $\mathrm{Bb}$-labeled cells did not extend as far caudally. This is in good agreement with our observations on the topography of the projection from the low IFS in the MGv to the low IFS in field A (see above).

These findings are also consistent with the results of experiment MSE042 (Fig. 3). Here, the HRP injections in field A were restricted to the ventral part of the $1-2-\mathrm{kHz}$ IFS, while in field DC, an injection of NY similar to that in the former case was done (see map in Fig. 3a). Compared with MSE039 (Fig. 6), the HRP cells do not extend as far rostrally, and they do not exactly meet the cloud of NY cells. Nevertheless, the continuity of the low-frequency band is apparent, and the caudal end of the NY array is again located dorsolaterally of the rostral end of the HRP band. We did not find any double-labeled cells in cases MSE042 and MSE050. 


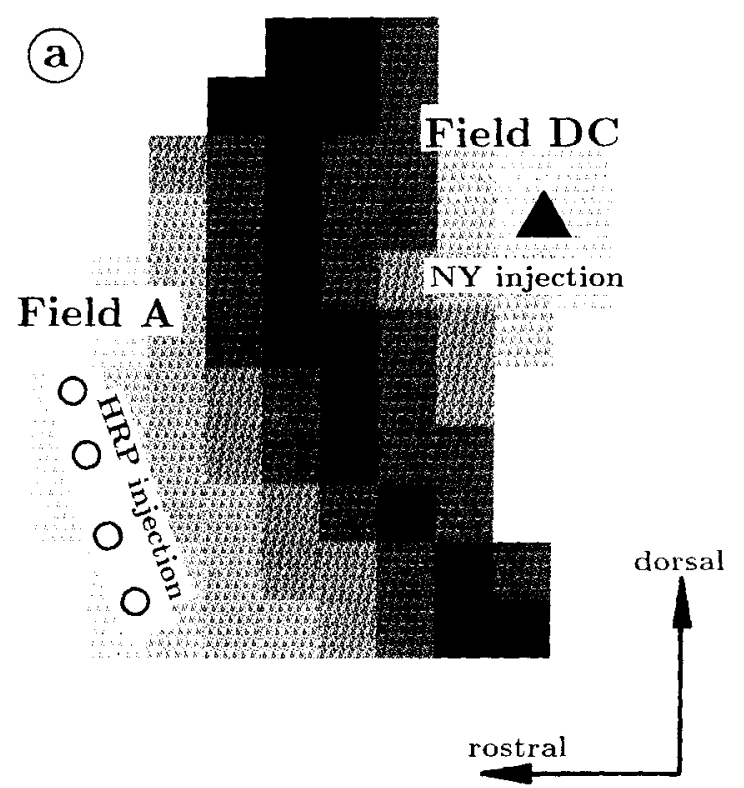

(b)

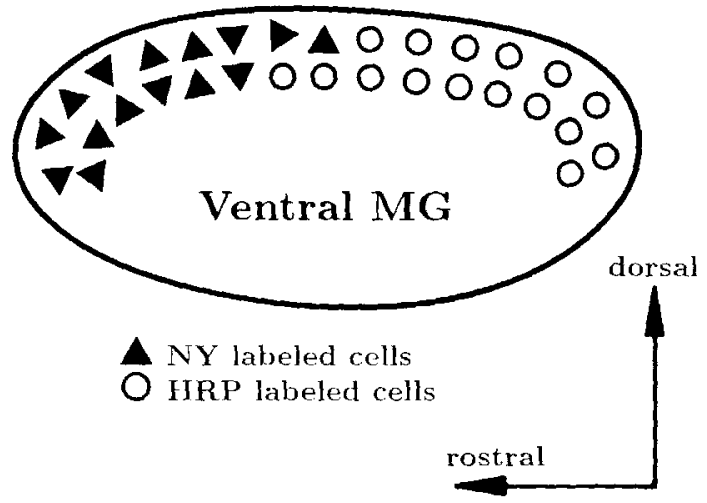

Fig. 11. Schematic representation of the results of experiment MSE039 (Fig. 6; see also MSE042, Fig. 3). a: Surface view of the auditory cortex (fields $A$ and DC). The BFs are represented by gray levels; light gray means low BFs and dark gray or black high BFs. The HRP and NY injection sites are represented by different symbols. b: Parasagittal section through the MGv. The cells projecting to field $\mathrm{A}$ are represented by circles and those projecting to field $\mathrm{DC}$ by triangles.

The results of these three experiments indicate that the thalamic cells innervating the low-frequency regions of fields $A$ and $D C$ constitute a single, continuous array of low-frequency cells in the $M G v$; the caudal part of this array projects to field $A$ and the rostral part to field $D C$. This is schematically depicted in Figure 11.

These experiments are also of interest for a comparison of the $\mathrm{MGcm}$ projection to fields $\mathrm{A}$ and DC. In Figure $6 \mathrm{~b}$, the histogram of the distribution of the differently labeled cells along the rostrocaudal extent of the $\mathrm{MGcm}$ is shown for case MSE039. Though the spatial scatter of the differently labeled cells is considerable, a weak topographic order of the projection can be recognized: Most MGcm cells projecting to the low BF site in field DC are located rostrally, whereas the cells projecting to the low IFS in field A tend to be located further caudally in the $\mathrm{MGcm}$. The cells projecting to the medium IFS of field $\mathrm{A}$ are situated between the former two populations.

\section{Afferents of the small field $S$}

A third tonotopic area is situated rostrally to field A. It occupies less than $1 \mathrm{~mm}^{2}$ of the cortical surface and was therefore named the "small field S" (Redies et al., '88). In two experiments, small amounts of HRP were injected into the small field. In case MSE038, the center of the injection site was in the high-frequency part of field S. In MSE049, the injection was placed more posteriorly, in an $8-\mathrm{kHz}$ location. The injection sites had a diameter of $300-400 \mu \mathrm{m}$ in both cases and thus did probably not extend beyond the borders of field $\mathrm{S}$.

The results of case MSE038 are shown in detail in Figure 12. Labeled cells were found medially of the MGv, in the rostromedial nucleus of the MG (MGrm). In other nuclei of the posterior thalamus, there were no labeled cells. Results were similar in MSE049. Most cells were concentrated in the MGrm, but in this case, a few scattered cells were found in the MGcm as well.

\section{Afferents of the nontonotopic auditory cortex}

The tonotopic fields are surrounded by areas which are acoustically responsive, but do not exhibit a tonotopic organization (Redies and Brandner, '87; Redies et al., '88).

Afferents of the ventrocaudal belt. A broad stripe of nontonotopic cortex, the ventrocaudal belt (VCB), is situated caudal to field $A$ and ventral to field DC. It extends for at least $2 \mathrm{~mm}$ from rostral to caudal and from dorsal to ventral. In five experiments, retrograde tracers were injected into the VCB. As a representative case, experiment MSE053 will be described in detail. Three HRP and three $\mathrm{Bb}$ injections were done in adjacent rows in the center of the ventrocaudal belt; the three HRP injections were situated caudal to the $\mathrm{Bb}$ injections (Fig. 13a). The diameters of the injection sites were about $500-800 \mu \mathrm{m}$ for $\mathrm{Bb}$ and about $300-700$ $\mu \mathrm{m}$ for HRP.

The $\mathrm{Bb}$-labeled cells in the MG were very numerous. A densely packed band begins about $600-700 \mu \mathrm{m}$ posterior to the rostral tip of the MG and extends for about $1,500 \mu \mathrm{m}$ caudally. The rostral two-thirds of this band appears in frontal sections as a continuous stripe of cells that surrounds the MGv dorsally, laterally, and ventrally (Figs. 13b, 14). Dorsally, the cell column is rather vast; laterally and ventrally, it becomes much narrower. In some sections, the continuity of the band was interrupted on the lateral side. In the caudal third of the band, the cells are less densely grouped, and a continuous band cannot be recognized here (Fig. 13b, section 31).

Because of its striking form, we name this structure the shell nucleus (see Patterson, '77). In Nissl-stained frontal sections, we could not identify a distinct cytoarchitectonic border between the shell nucleus and the MGv or the MGrm. Possibly, the transition between the MGs and these two nuclei is gradual; i.e., the respective cell populations intermingle to a certain extent. The MGs seems to extend somewhat into the fiber tracts surrounding the MG: laterally and ventrally, many labeled cells were found interspersed in axon bundles. A second population of Bb-labeled cells was observed in the MGcm. The labeled MGcm cells 
(a)

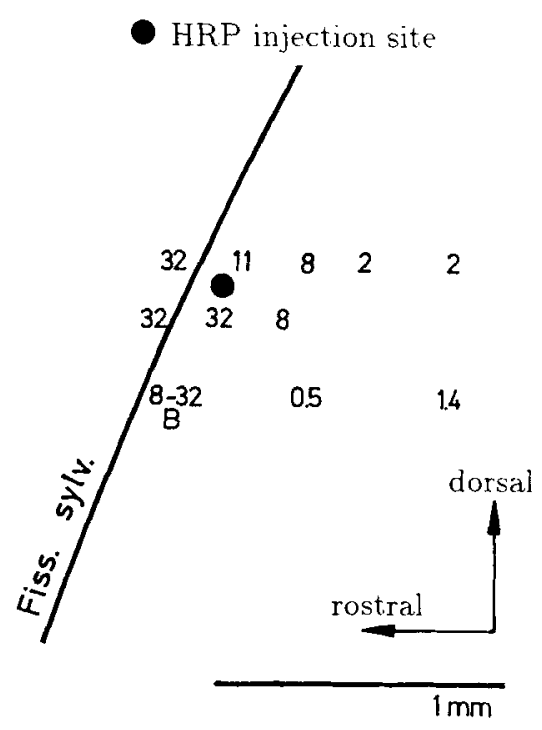

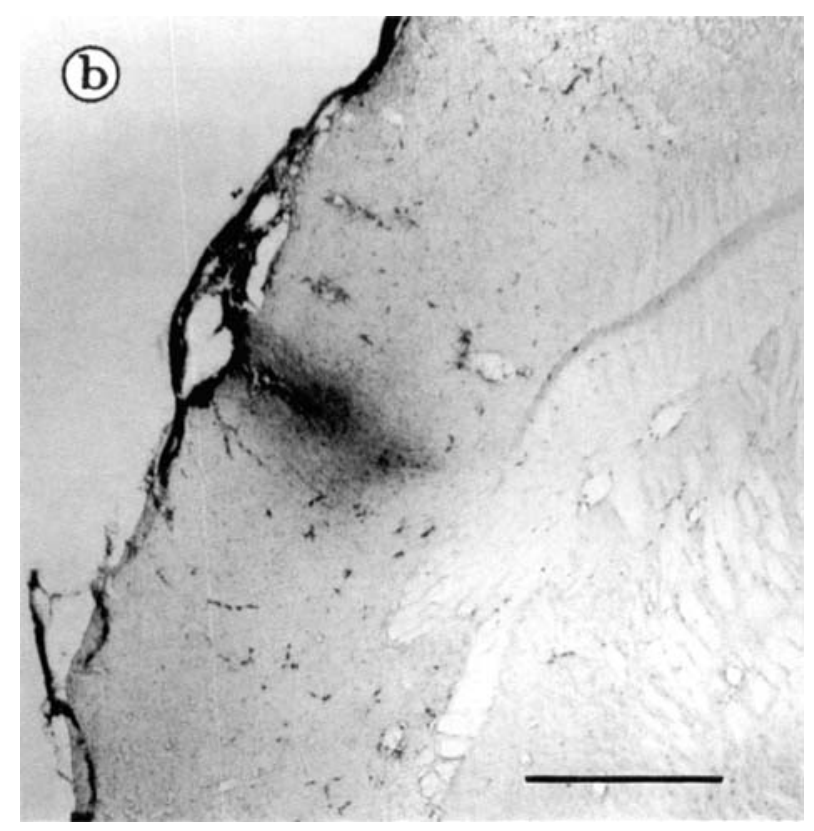

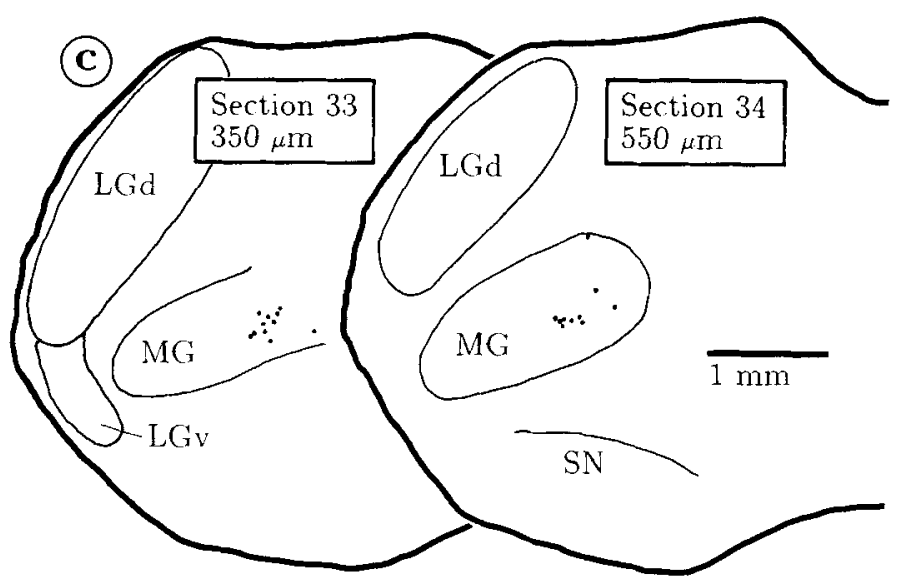

Fig. 12. Experiment MSE038. a: The BF map of MSE038. HRP was injected into field $S$. b: Photomicrograph of the center of the HRP injection site. The section is not counterstained. Calibration bar $1 \mathrm{~mm}$. c:
Frontal sections through the posterior thalamus. Each dot represents one HRP-labeled cell. See Figure 3 for further explanations.

tered over a large nuclear volume, comparable to that of the above-described cases. This indicates that one point in the VCB receives afferents from many points in the shell nucleus; i.e., there is a high degree of convergence/divergence in the projection from the shell nucleus to the VCB. Again, some labeled cells were observed in the MGcm.

Afferents of the rostral belt (RB). Dorsally, laterally, and ventrally, the small tonotopic field $S$ is surrounded by a narrow stripe of nontonotopic cortex named the rostral belt (RB) (Redies et al., '88). In two animals, small amounts of $\mathrm{Bb}$ were injected into the $\mathrm{RB}$. In both cases, the diameter of the injection site did not exceed $300 \mu \mathrm{m}$. Nevertheless, because of the small extent of the rostral belt, a diffusion of tracer into the neighbouring cortical areas (field $\mathrm{S}$ and somatosensory areas) cannot be excluded; therefore, the present results have a preliminary character, and will only 


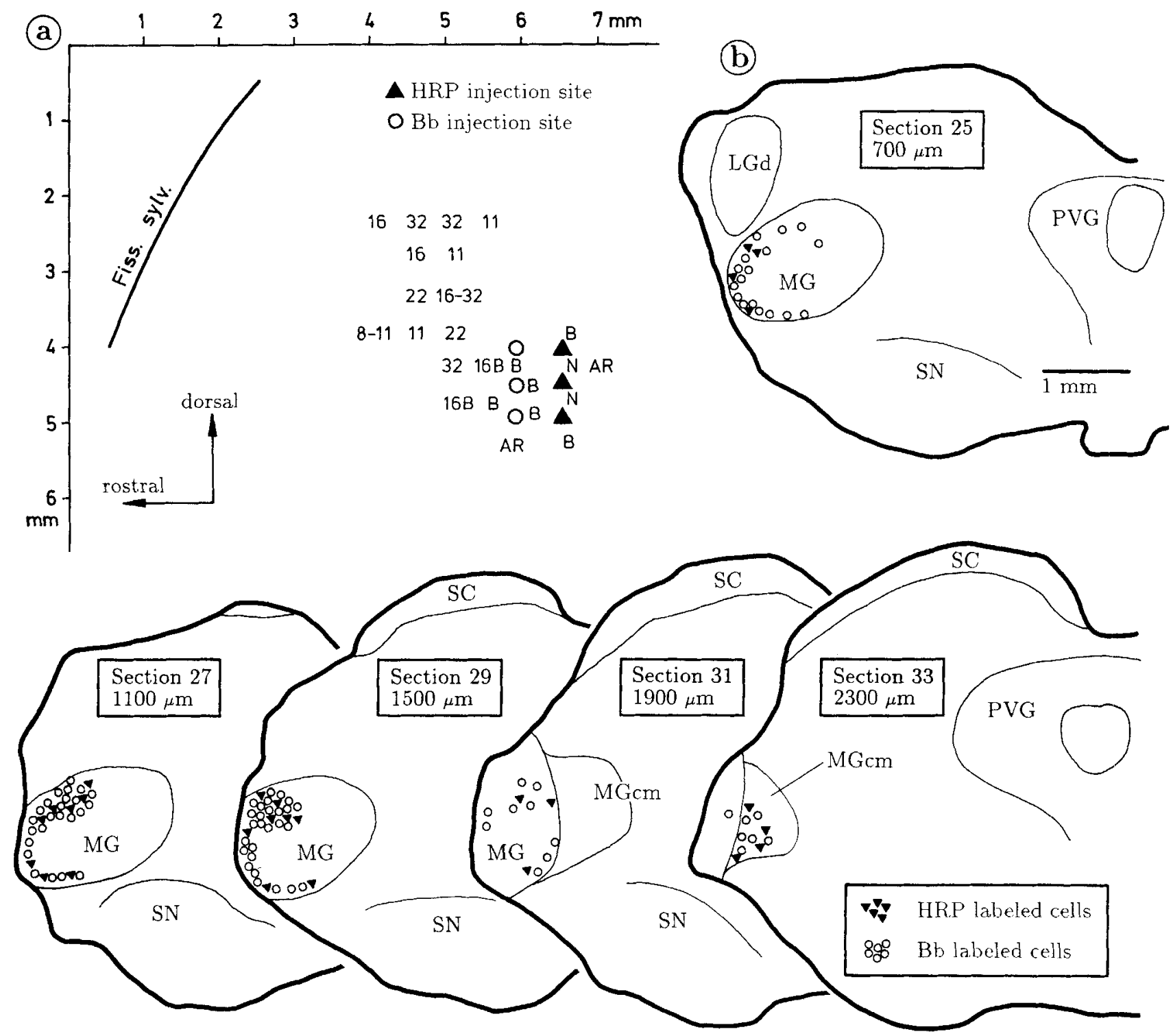

Fig. 13. Experiment MSE053. a: The BF map of MSE053. Bb was injected into three sites rostrally in the VCB and HRP into three loci situated more caudally. b: Frontal sections through the posterior thala-

be briefly sketched. In MSE049, we injected Bb ca. $200 \mu \mathrm{m}$ rostrally to field S. Labeled neurons were observed medial to the rostral pole of $\mathrm{MG}$, and more rostrally, in the ventrobasal complex VBC. The labeled cells were arranged in a narrow column with a rostrocaudal extension of about $600-$ $800 \mu \mathrm{m}$. In MSE048, the injection site was situated more ventrally than in MSE049. Again, labeled neurons were found dorsomedial to the rostral pole of the MG and in the VBC.

\section{DISCUSSION}

In this study, we determined the subnuclei of the auditory thalamus that project to the tonotopic fields in the guinea pig auditory cortex. In addition, the thalamic afferents to a nontonotopic area, the ventrocaudal belt, were investigated, and preliminary data are provided for the rostral belt. mus. Each empty circle stands for three to ten Bb-labeled neurons and each filled triangle for one to three HRP cells.

The anterior (A) and the dorsocaudal (DC) tonotopic fields (Redies et al., '88; see also Hellweg et al., '77) receive a strong and topographically ordered projection from the ventral nucleus of the MG. The caudomedial nucleus ( $\mathrm{MGcm}$ ) also innervates both areas, but this projection is weaker, and the topography is less strict. In some animals, a few scattered labeled cells were also found in a region dorsomedial to the MGv.

The afferents to the small field $\mathbf{S}$ come mainly from the MGrm, a nucleus situated medially to the MGv in the rostral half of the MG (Fig. 2a). In one of the two experiments in which field $\mathbf{S}$ was injected, labeled cells were also found in the MGcm.

After injection of tracer into the ventrocaudal belt, labeled cells were found dorsal, lateral, and ventral to the MGv. The labeled cells form a continuous band that sur- 


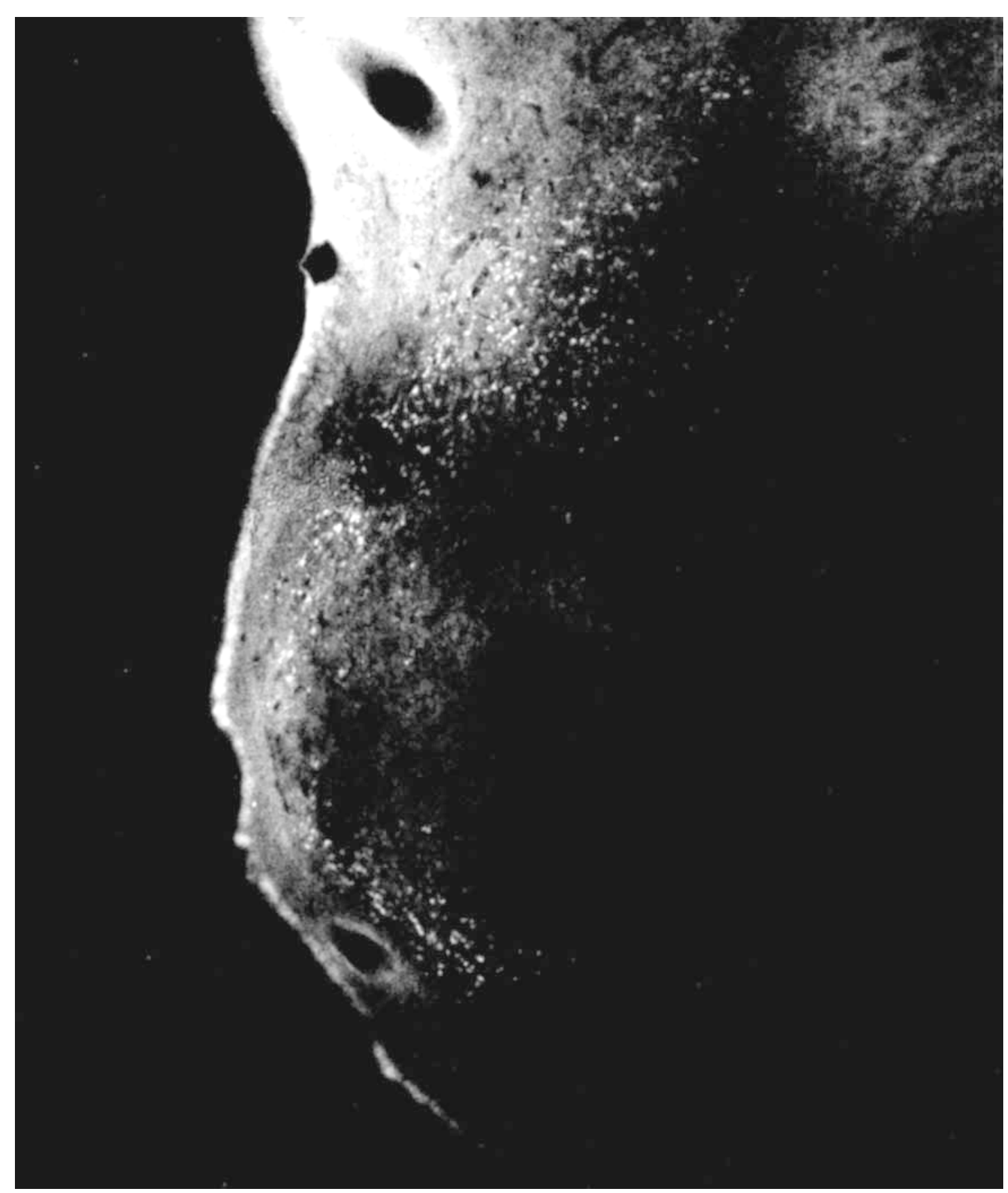

Fig. 14. Photomicrograph of Bb-labeled cells in the shell nucleus, section 27 from experiment MSE053. Magnification 96:1.

rounds the MGv like a shell. A second population of labeled neurons was found in the $\mathrm{MGcm}$. Preliminary results indicate that the rostral belt receives afferents from an area situated dorsomedially in the rostral pole of the $\mathrm{MG}$ and continuing into the ventrobasal complex (somatosensory thalamus).
The projection from the MGv to field A

The cell clouds labeled after injections into low and medium IFSs of field A resembled a sphere or rod, and discontinuities in the cell masses were not apparent. It was only after injections into high BF sites that slight discontinuities 


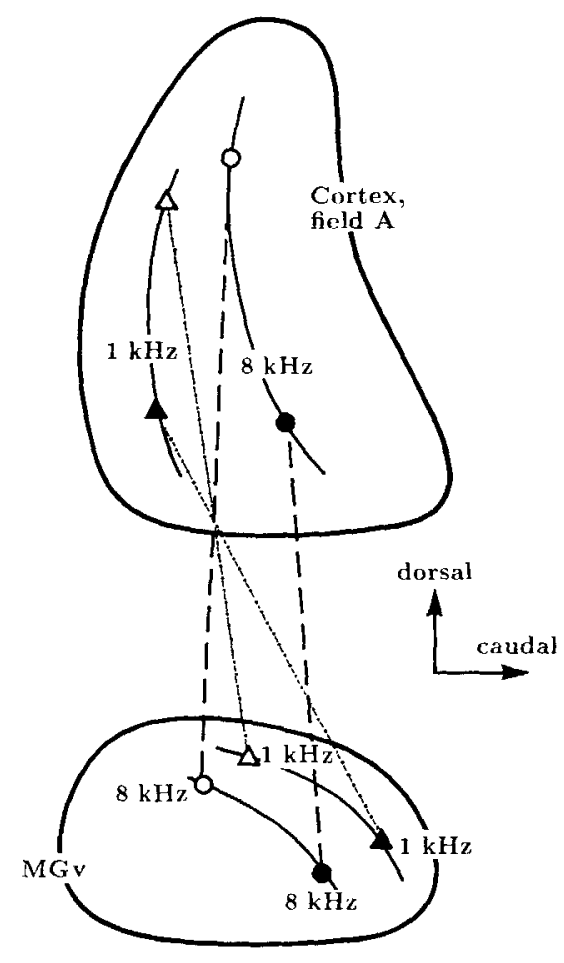

Fig. 15. Schematic representation of the projection from the MGv to field $\mathrm{A}$. The figure illustrates that there is a topographic organization along the frequency axis and along the isofrequency dimension. See text for further explanations.

were observed in some, but not all, animals. Similar differences between the low- and high-frequency parts of the auditory thalamocortical system have been observed in the cat, and it has been hypothesized that they are related to differences in binaural information processing for high and low frequencies (Andersen et al., '80; Middlebrooks and Zook, '83).

Presumably, the BF of the labeled cells in the MG equals the BF of the cortical injection site, and we can therefore reconstruct the general outlines of tonotopy in the MGv. An evaluation of the experiments where field $A$ was injected yieids the following picture of frequency representation in the corresponding part of the MGv. High frequencies are located rostrally in the MGv. The medium frequencies lie somewhat more caudally, and the isofrequency bands are inclined relative to the sagittal plane: They proceed from rostromedial to caudolateral. The low-frequency bands lie caudodorsomedially of the medium-frequency cells (see Fig. 6) and are limited to the posterior half of the MGv. In other words, in that portion of the MGv which innervates field $\mathrm{A}$, best frequencies become progressively higher when proceeding from caudal to rostral, but also when proceeding from dorsomedial to ventrolateral. This is schematically depicted in Figure 16b-d. The drawings show reconstructions of tonotopy in the frontal plane. In a recent electrophysiological mapping study of the guinea pig $\mathrm{MG}$, these findings were confirmed (Redies, to be published).

Topographically, the projection from the MGv to field A undergoes an inversion in that rostral sites in the MGv project to caudal parts of $\mathrm{A}$ (high frequencies), whereas inter-

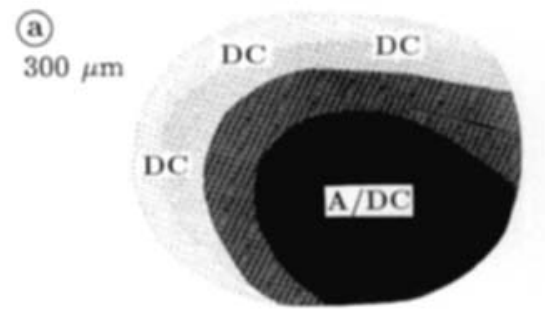

(b) $800 \mu \mathrm{m}$
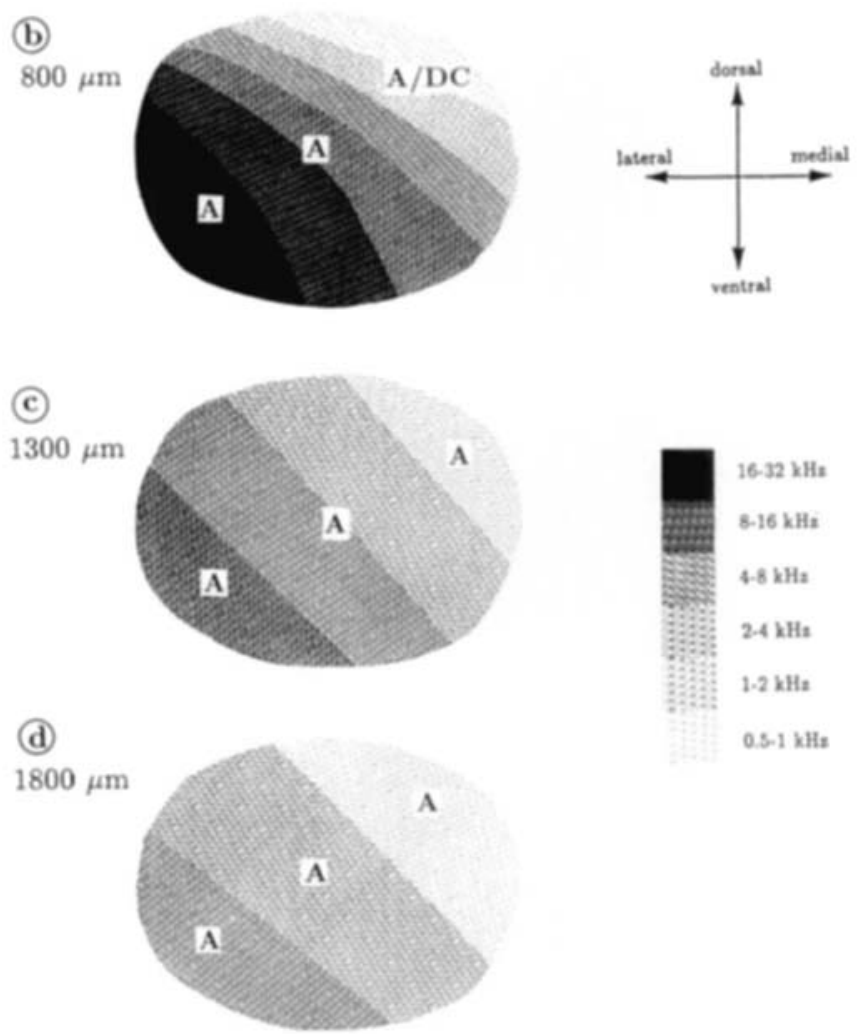

Fig. 16. The tonotopy in the MGv is shown in a frontal perspective for four different levels arranged from rostral (a) to caudal (d). The distance of each "section" relative to the rostral pole of the MG is indicated beside each drawing. The $\mathrm{BF}$ are coded by gray levels; see scale to the right. The labels " $A$ " and "DC" indicate the cortical target fields of the ascending projections. "A/DC" in a and b means that the neuronal populations projecting to the two tonotopic fields overlap here or that our results are not detailed enough for a more precise statement. See text for further explanations.

mediate and caudal sites in the MGv project to more rostral portions of $\mathrm{A}$ (medium and low frequencies). There is also a topographic gradient parallel to the isofrequency contours: the dorsal portions of a cortical IFS receive afferents from rostral parts of the corresponding thalamic isofrequency band; more ventral portions of a cortical ISF are innervated by more caudal parts of the thalamic band. This is schematically depicted in Figure 15 for the low (cf. Fig. 4) and medium (cf. Figs. 5, 6) frequencies. However, we are not sure whether this or a similar topographic gradient also exists in the high-frequency part of the projection (see Results and Fig. 7), and therefore, the high frequencies are omitted in Figure 15 . 


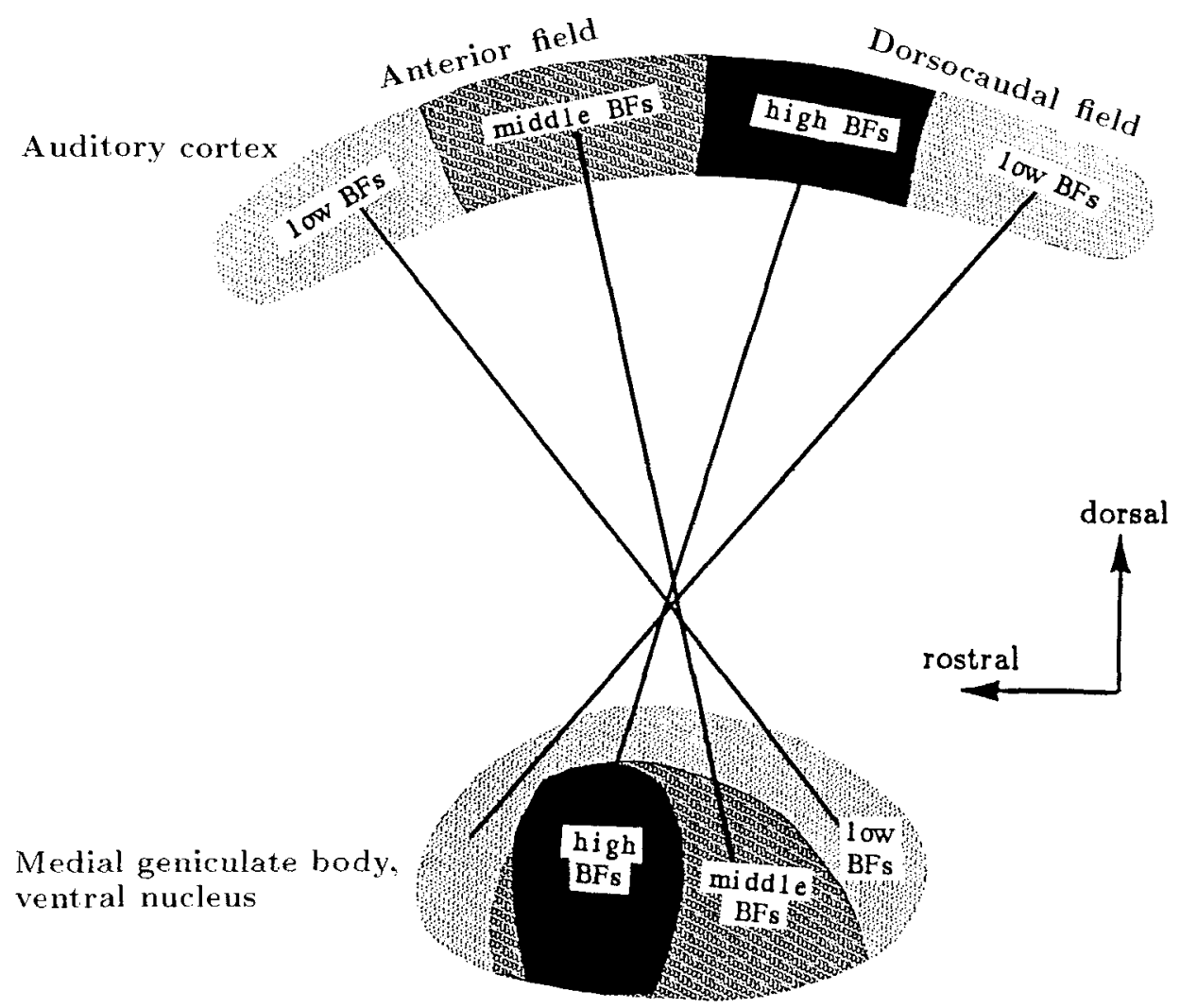

Fig. 17. Summary of the projection from the MGv to fields A and $\mathrm{DC}$. The BFs in the thalamus and cortex are represented by gray levels; the lines connecting the MGv to the cortex represent the fiber tract from the MGv to fields $A$ and DC as described in the text.
Figure 15 also illustrates that the ascending fiber tract undergoes an inversion over the rostrocaudal axis. Similar inversions exist in the projection from the MG to the primary auditory cortex of the rhesus monkey (Mesulam and Pandya, '73) and in other sensory systems, e.g., in the thalamocortical somatosensory fiber tract (e.g., Bernardo and Woolsey, '87; review in Creutzfeldt, '83).

\section{The projection from the MGv to field DC}

The cells in the MGv projecting to field DC are spatially segregated from the cells projecting to field A. Roughly speaking, our results show that field DC is innervated by the rostral part of the $\mathrm{MGv}$, whereas the thalamic cells projecting to field A are located more caudally (Figs. 16, 17).

The labeled cell bands observed after injection into field $\mathrm{DC}$ are curved around the lateral margin of the $\mathrm{MGv}$ in the very rostral tip (the first $300-400 \mu \mathrm{m}$ ) of this nucleus and become more stretched further caudally (see schematic drawing in Fig. 16 a,b and, e.g., Figs. 3c, 6c, 8c, 9, 10b). The topography of the connection between the MGv and field $D C$ is therefore more difficult to describe. Seen from an imaginary center in the rostral $\mathrm{MGv}$, the cell bands projecting to the low-frequency part of DC are located outside (i.e., ventrally, laterally and dorsally) of the cell bands innervating the high-frequency part of DC. This is particularly clear in animal MSE051, where different tracers were injected into the high-frequency and low-frequency parts of DC, respectively (Fig. 10; see also Fig. 3c).
The tonotopy in the rostral part of the MGv may be summarized by Figure 16a: the outer isofrequency bands resemble a set of nested crescents. Light gray stands for low BFs and dark gray for higher BFs. The cells representing the highest frequencies (black) appear as a sphere at the center (cf. Fig. 3c). Experiments MSE042 and MSE051 (Figs. 3, 10) show that there is a very abrupt transition from high to low frequencies without intermediate values in the rostral pole of the MGv. This fits exactly the tonotopic structure of field $\mathrm{DC}$, which is innervated by this part of the MGv: in our cortex-mapping experiments, we consistently found an abrupt skip from high to low BFs in the dorsal half of field DC; the intermediate frequencies were represented ventrally in field DC only and confined to a very limited area (e.g., Figs. 3a, 8a, 10a; see Redies et al., '88, for details).

\section{Different parts of the thalamic tonotopic map are projected onto different cortical fields}

Though the geometry of the isofrequency contours is different in the rostral and caudal parts of the MGv, there is only a single, continuous tonotopic map in the MGv. This became particularly obvious in experiments where different tracers were injected into sites with like BFs in fields $A$ and DC, respectively. In case MSE039 (Fig. 6), for example, the $1-2-k H z$ sites were injected in fields A (HRP) and DC (NY). The labeled cells in the MGv build one continuous array, which runs from the caudal to the rostral pole of the MGv. The rostral part of this array innervates the low IFS in field DC; the caudal part innervates the low IFS in field A. We would emphasize that the continuity of the NY and HRP 
cells is apparent though the tracers were injected in cortical sites very distant from each other: the low IFSs are located at the extreme rostral and caudal ends of fields A and DC.

In conclusion, our observations suggest a topography of the projection from the $M G v$ to fields $A$ and $D C$ as schematically shown in Figure 17. The drawing stresses the fact that there is only one continuous representation of frequency range in the MGv but that there are two such representations in the auditory cortex innervated by the MGv. Our results indicate how this "duplication" of the cochlear image between thalamus and cortex comes about: As a matter of fact, the thalamic map is not duplicated, but split into two parts: the rostral portion of the map is projected onto field DC and the caudal portion onto field $A$.

\section{The parcellation of the guinea pig MG}

The literature about the guinea pig MG is quickly summarized, since this structure has received little attention in the past. On cytoarchitectonic grounds, Gerebtzoff and Wauters ('41) divided the guinea pig MG into a ventral, a dorsal, and a medial part ("noyau profond"). However, their medial nucleus is located dorsal to the $\mathrm{MGv}$, and not, as, e.g., in the cat, medially (Morest, '64). Völker and Graef ('65, '66) recognize two nuclei: rostrally in the MG, they describe a lateral cell mass with high cellular density and a medial part which is less densely packed. Further caudally, the medial nucleus disappears (see also Cajal, '09; Hess, '55).

The present results make it possible to define the subdivisions of the MG not only according to the classic cytoarchitectonic criteria such as cellular packing density, soma morphology, etc., but also on the basis of the cortical projections of the nucleus. Our conclusions differ somewhat from the above-cited studies (which are, anyhow, not unanimous).

1. The MGv extends over the rostral two-thirds of the MG (Fig. 2a,b). It projects in a strictly topographic fashion to the tonotopic cortex. There is only one tonotopic map in the MGv, and we therefore consider it as a single nucleus. It is interesting in this context that so far, functional differences between the fields A and DC, which are the cortical target of the MGv efferents, have not been found (see Redies et al., '88).

2. The shell nucleus (MGs) is defined here as the region that provides the main input to the (nontonotopic) ventrocaudal belt (Figs. 2a,b, 14). The MGs surrounds the ventral MG dorsally, laterally, and ventrally. We found it impossible to define clear cut cytoarchitectonical boundaries be tween MGs and MGv and between MGs and MGrm. The dorsal part of the shell nucleus might partly correspond to the medial nucleus of Gerebtzoff and Wauters, but neither these authors nor Völker and Graef mention anything comparable to the striking shell form described in the current paper.

3. The rostromedial nucleus (MGrm) lies medially in the rostral part of the MG (Fig. 2a). It appears to correspond to the medial cell mass of Völker and Graef ('65, '66, see above). We found labeled cells in the MGrm only after injections of tracer into field $\mathrm{S}$. The MGrm is relatively voluminous compared with the small size of field S; possibly, it projects also to other (not necessarily auditory) cortical fields not investigated in the present study, or it consists of several subnuclei which are not distinctly apparent in our Nissl material.

4. The caudomedial nucleus (MGcm) is a cell mass in the caudal half of the MG (Fig. $2 \mathrm{~b}-\mathrm{d}$ ). It can easily be distin- guished from the other parts of the MG by its large, deeply staining cells. It is therefore surprising that it was not described by Gerebtzoff and Wauters ('41) or Völker and Graef ('65, '66). Possibly, these authors, although recognizing the nucleus as a distinct entity, did not include it into the MG. The MGcm sends fibers to all cortical auditory fields we investigated, with the possible exception of the rostral belt region (see Results). The projection is typically rather sparse with only a weak tendency for a topographic or tonotopic organization.

\section{Comparison with other "primitive" mammalian species}

A ventral nucleus of the MG, with similar cytoarchitecture and location to the guinea pig, MGv has been described in rat, tree shrew, bats, and other species (see Winer, '85, for review). There is little doubt that the ventral nucleus is a homologue structure common to all mammals. The projections of the $\mathrm{MGv}$ to the auditory cortex were investigated in the rat by Patterson ('77) and Scheel ('87). Both authors agree that the MGv projects in a strictly topographic fashion to the primary auditory cortex (defined by cytoarchitectonic means). In the tree shrew Tupaia glis, similar observations were made (Oliver and Hall, '78a,b).

Are there structures equivalent to the guinea pig shell nucleus in other species? Patterson ('77) in his analysis of the rat MG follows more or less closely the well-known scheme proposed by Morest ('64) for the cat. Patterson delimits in the rat MG a dorsal, a marginal, and a ventrolateral nucleus, situated dorsal, lateral, and ventral to the MGv, respectively. Additionally, he identifies a caudal nucleus located posterior to the MGv. A similar, though not identical scheme was proposed by Ledoux et al. ('85, '87; see also Gurdjian, '27; Winer and Larue, '87). According to Patterson ('77), these four subnuclei project to the auditory belt cortex (defined by cytoarchitectonic criteria). The projection is topographically organized, i.e., the different subnuclei project to different parts of the auditory belt. However, "the connectional data suggest that ... the dorsal division, the marginal zone, the ventrolateral nucleus and the caudal division are all part of the same cortical projection system" (Patterson, '77, p. 33). Consequently, Patterson proposes to label this set of subnuclei the "shell nuclei."

Topographically, the shell nuclei of the rat appear to correspond roughly to the guinea pig shell nucleus; moreover, they exhibit similar connections with the auditory cortex. A difference between guinea pig and rat may be that in the rat, the different components of the shell project topographically to different parts of the belt, while in the guinea pig, the entire shell nucleus projects to the ventrocaudal belt region. But we did not investigate the thalamic projections to the dorsocaudal belt and the region just caudal to field $\mathrm{DC}$, and it is therefore premature to draw definite conclusions.

In the MG of the opossum, several authors have described two major subdivisions: a central nucleus is surrounded dorsally, laterally, and ventrally by a marginal nucleus (e.g., Bodian, '39; Oswaldo-Cruz and Rocha-Miranda, '68). This scheme ressembles-at least topographically - that of the MGs enclosing the MGv in the guinea pig.

Thus, it is possible that the guinea pig shell nucleus is not a structure unique to this species but a typical feature of MG organization in rodents, and maybe also in marsupials 
and other mammalian orders. The idea that a shell nucleus is part of the basic plan of MG organization in Mammalia merits, in our opinion, further investigation.

Our description of the MGrm and MGcm closely resembles the analysis of the MG in the tree shrew Tupaia glis by Oliver and Hall ('78a,b). These authors too subdivide the medial part of the MG into a rostral and a caudal subnucleus. The rostral subnucleus of the tree shrew consists of cells which are larger and less densely packed than those in the ventral nucleus; it projects to a single cortical field (named field B by Oliver and Hall) situated dorsal to the primary auditory cortex. The caudal nucleus of the tree shrew MGm consists of large and deeply stained cells and projects to the entire auditory cortex. These parallels between tree shrew and guinea pig suggest that at least the magnocellular nuclei in both species are homologous. It is more problematic to make this assumption for the rostromedial nuclei, since the relations between field S (guinea pig) and field B (tree shrew) are not clear.

In contrast to this, in the rat, the medial part of the MG, which extends over the whole rostrocaudal length of the MG, has not been further subdivided (Patterson, '77; LeDoux et al., '85, '87; Winer and Larue, '87). The literature contains contradictory descriptions about the ascending projections of this subnucleus (Patterson, '77; Scheel, '87), and a detailed comparison of rat and guinea pig in this respect is at present not possible.

The above discussion remains unsatisfying because the available data are sparse and because it is sometimes uncertain whether the apparent differences between species are real or due to the use of different methods. The conclusions of this paper are largely based on the projections from the MG to physiologically identified cortical fields, but a corresponding project has not yet been conducted in another "primitive" mammalian species. The state of knowledge is more advanced in the cat, and the last paragraphs of this paper focus on a comparison of the guinea pig with this latter species.

\section{Comparison with cat: afferents of the tonotopic fields}

In the cat cortex, four tonotopically organized auditory fields have been distinguished (Reale and Imig, '80). Two of these, the anterior auditory field (AAF) and the primary auditory field (AI), are similar in many physiological aspects to areas $\mathrm{A}$ and DC in the guinea pig, though differences, especially in the layout of tonotopy, have also been noted (see Redies et al., ' 88 , for discussion). The thalamic nuclei projecting to AI and AAF have been investigated by several workers in the past (for AI see, e.g., Winer et al., '77; Niimi and Matsuoka, '79; Andersen et al., '80; Middlebrooks and Zook, '83; Mitani and Shimokouchi, '85; for AAF see Andersen et al., '80; Imig and Morel, '84; Morel and Imig, '87; for review see Imig and Morel, '83). We shall focus here on the work of Andersen et al. ('80) and Morel and Imig ('87), since these authors employed methods similar to those of the present project; i.e., they combined electrophysiological mapping and tract-tracing experiments in the same animals.

Andersen et al. ('80) injected one or two tracers into sites in $\mathrm{AAF}$ and/or AI with known BF and then determined the thalamic nuclei connected to these fields and the fine topographic structure of the projection. After injection into AI, they found two arrays of labeled cells, a medial and a lateral one, in the auditory thalamus. The lateral column is located mainly in pars lateralis and pars ovoidea of the ventral division of the MG (see Morest, '64; and Winer, '85, for subdivisions of the cat $M G$ ). The medial column begins in the lateral nucleus of the posterior thalamus (POl) and then continues caudally. It traverses the deep dorsal nucleus (DD) and ends in the medial (magnocellular) nucleus of the MG. The projections from both columns are topographically organized, but the topographic order is stricter in the lateral than in the medial column. The thalamic afferents of AAF are similar to those of AI, except that AAF gets a much stronger input from $\mathrm{POI}$ and less afferents from the MGV (see below).

Thus, cat and guinea pig appear similar in some essential features of the auditory thalamocortical system: AI/AAF (cat) and A/DC (guinea pig) receive both a strong and topographically ordered projection from the tonotopic auditory thalamus. Also, in both species, there is a second projection which originates (partly) in a magnocellular subnucleus of the MG. But a detailed comparison reveals also substantial interspecies differences.

The structure of the $M G$. The lateral cell column of the cat as defined by Andersen et al., which is located in the ventral division of the MG, might correspond to the cell column we found in the guinea pig MGv. These nuclei are similar in both species in cytoarchitectonics, location, and response characteristics (cat: see, e.g., Aitkin and Webster, '72; Calford, ' 83 ; Winer, '85; guinea pig: Redies, to be published). But the medial column of labeled cells in the cat, which is located in POI, DD, and MGm, is obviously different from the population of cells in the guinea pig's $\mathrm{MGcm}$. In the cat, the column extends over three different nuclei, while in the guinea pig, it is confined to the MGcm. Moreover, it is not quite clear whether the magnocellular nuclei in cat $(\mathrm{MGm})$ and guinea pig $(\mathrm{MGcm})$ are equivalent. On the one hand, the magnocellular nuclei innervate the entire auditory cortex in both species and are cytoarchitectonically similar; i.e., both contain many large and deeply staining neurons (cat: see Winer, '85, for review). On the other hand, the location of the nuclei is different in both species. The MGcm in the guinea pig lies in the caudal half of the MG, while the MGm in the cat is situated in the rostral three-fourths of the MG (Berman and Jones, ' 82 ; Winer, '85).

From a purely topographical point of view, the region occupied in the cat by $\mathrm{MGm}, \mathrm{DD}$, and $\mathrm{POl}$ might roughly correspond to the guinea pig's MGrm (compare, e.g., Plates 84-87 in Berman and Jones, '82, with Fig. 2a). But the only tonotopic cortical field that receives input from the MGrm is the small field $\mathrm{S}$. We are not aware of any literature report describing something similar to field S and the MGrm in the cat.

The fine structure of the projection. In the cat, the neurons projecting to sites of like BFs in AI and AAF occupy identical positions in the MGv and in $\mathrm{POl}$, though the MGv projects heavier onto $\mathrm{AI}$ and $\mathrm{POl}$ heavier onto AAF (Andersen et al., '80; Morel and Imig, '87). The present paper shows that in the guinea pig, the cells in the MGv projecting to sites of like BF in fields $A$ and DC are spatially segregated (Fig. 11), although the two populations may slightly overlap in a limited portion of the thalamic isofrequency band (cf. Fig. 6c, section 32).

One might speculate that in the cat, fields AAF and AI are parallel information processors in the sense that information from the same thalamic sites is to some degree processed simultaneously by $A I$ and AAF (Morel and Imig, 

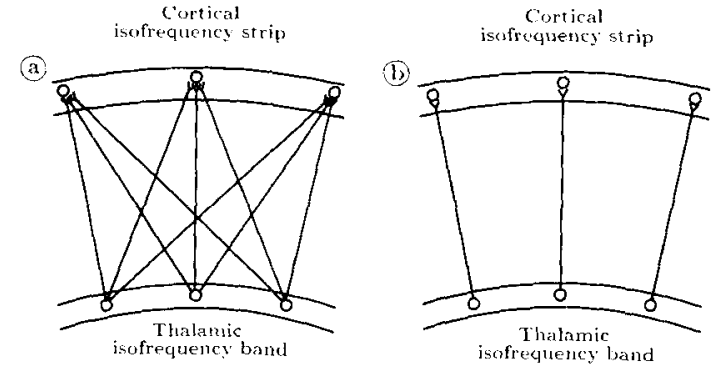

Fig. 18. a: The schema illustrates that in the cat, each sector of an isofrequency strip in AI apparently receives input from the entire thalamic band of corresponding BF. b: In the guinea pig, the projection from the MGv to field A is organized in a point-to-point fashion. See text for further explanations.

'87). Our results show that this hypothesis is certainly not valid for the guinea pig. However, the functional meaning of this interspecies difference is at present unclear.

There is still another aspect of the fine structure of the thalamocortical projection in which cat and guinea pig seem to differ. Andersen et al. ('80) reported that in the cat, different sectors of a cortical IFS in AI or AAF receive afferents from the same thalamic regions. "Since multiple injections along isofrequency contours in AAF or AI produced the same general ... labeling in the $\mathrm{MG}$ as was obtained with single injections, it is probable that all sectors along an isofrequency contour in cortex are interconnected with the same isofrequency contours in the MG" (Andersen et al., '80, p. 698; see also Colwell, '77; cited from Andersen et al., '80). This concept of a "divergence/convergence" projection, schematically illustrated in Figure 18a, has meanwhile been refined by Middlebrooks and Zook ('83). According to these workers, different cortical EI-bands (e.g., a ventral and a more dorsal one) receive afferents from the same thalamic EI-bands. Different cortical EE-bands ${ }^{1}$ are innervated by thalamic populations that largely, though not completely, coincide.

In contrast, in the guinea pig, we observed a "point-topoint" projection rather than a "divergence/convergence" pattern, at least for the low- and medium-frequency part of the projection. Schematically, this finding is represented in Figure $18 \mathrm{~b}$ (see also Fig. 4). A possible functional meaning of this difference between cat and guinea pig can be illustrated by the following example. Suppose that there is an ordered representation (or map) of an acoustic parameter in the MG which coexists with the isofrequency planes in this nucleus (e.g., best modulation frequencies for amplitude-modulated signals; see Hose and Langner, '87; and Langner and Schreiner, '87). A projection from the MGv to the cortex, if organized as in Figure 18b (guinea pig), could transmit such a map. We would even expect it in the cortex if it existed in the MG. On the other hand, the structure illustrated in Figure 18a (cat) would hardly be appropriate to transmit any ordered gradient contained in the isofrequency planes from lower to higher brain levels but might instead underlie the formation of a new "computational" map.

${ }^{1} \mathrm{EE}$ stands for excitatory-excitatory and is generally used to characterize auditory cells that are driven by acoustic input to the contralateral and ipsilateral ear. EI means excitatory-inhibitory and refers to cells that are excited by contralateral and inhibited by ipsilateral stimulation. See Irvine ('86) for a discussion of these notions.

\section{Comparison with cat: afferents of the nontonotopic fields}

It is difficult to compare the thalamic afferents of the ventrocaudal belt (VCB) in the guinea pig with the cat, since it is not clear which field in the cat is the analog (and/or homo$\log$ ) of the VCB. The most likely candidate is perhaps the secondary auditory cortex (AII). In VCB (guinea pig) and AII (cat), there is no (or only a weak) tonotopic grandient, and the cells have, compared to the tonotopic cortex, longer latencies and broader tuning curves. Also, both fields have a similar location relative to the tonotopic fields: AII in the cat is situated ventral to AI, and VCB in the guinea pig is located ventral to field DC; i.e., both areas occupy the region ventral to the more caudally placed tonotopic field (cat: see, e.g., Andersen et al., '80; Schreiner and Cynader, '84; guinea pig: see Redies et al., '88, and the map in Fig. 13a).

The thalamic nuclei projecting to AII (cat) and to VCB (guinea pig) exhibit some similarities. AII receives afferents from the MGd and the MGm (Winer et al., '77; Niimi and Matsuoka, '79; Andersen et al., '80). In addition, a projection from the ventrolateral nucleus (VL) has been described (Andersen et al., ' 80 ). The cat's MGd and VL correspond topographically approximately to the dorsal and ventral parts of the guinea pig's shell nucleus (for MGm and MGcm see discussion above).

A structural difference between both species seems to be that there is a continuous shell nucleus in the guinea pig, while the dorsal and the ventrolateral nuclei of the cat $\mathrm{MG}$ are spatially separate. However, this difference might be of minor functional importance: the cat's VL and MGd are very similar in neuronal morphology and response characteristics, and consequently, it has been proposed that VL should be considered as part of the dorsal division (Winer, '85).

\section{ACKNOWLEDGMENTS}

The authors would like to thank Dr. Klaus Albus, Dr. Ulrich Sieben, and Dr. Petra Wahle for comments on an earlier version of this paper and Sabine Geisendorf, Susanne Lausmann, Eva Niksch, and Ursula Steveling for excellent. histological assistance.

\section{LITERATURE CITED}

Adams, J.C. (1980) Stabilizing and rapid thionin staining of TMB-based reaction product. Neurosci. Lett. 17:7-9.

Aitkin, L.M., and W.R. Webster (1972) Medial geniculate body of the cat: Organization and responses to tonal stimuli of neurons in ventral division. J. Neurophysiol. 35:365-380.

Andersen, R.A., P.L. Knight, and M.M. Merzenich (1980) The thalamocortical and corticothalamic connections of AI, AII and the anterior auditory field (AAF) in the cat: Evidence for two largely segregated systems of connections. J. Comp. Neurol. 194:663-701.

Berman, A.L., and E.G. Jones (1982) The Thalamus and Basal Telencephalon of the Cat. A Cytoarchitectonic Atlas With Stereotaxic Coordinates. Madison: The University of Wisconsin Press.

Bernardo, K.L., and T.A. Woolsey (1987) Axonal trajectories between mouse somatosensory thalamus and cortex. J. Comp. Neurol. 258:542-564.

Bodian, D. (1939) Studies on the diencephalon of the virginia opossum. Part I. The nuclear pattern in the adult. J. Comp. Neurol. 71:259-323.

Cajal, S.R. (1909) Histologie du Système Nerveux de I'Homme et des Vertébrés. Tome II. Paris: Maloine (reprinted by C.S.I.S., Madrid, 1972.) 
Calford, M.B. (1983) The parcellation of the medial geniculate body of the cat as defined by the auditory response properties of single units. J. Neurosci. 3:2350-2364.

Colwell, S.A. (1977) Reciprocal Structure in the Medial Geniculate Body. Thesis, University of California, San Francisco.

Creutzfeldt, O.D. (1983) Cortex Cerebri-Leistung, strukturelle und funktionelle Organisation der Hirnrinde. Berlin: Springer Verlag.

Gerebtzoff, M.A., and V.S. Wauters (1941) Recherches sur l'ecorce cerebrale et le thalamus du cobaye. II. Systematisation cortico-thalamique et voies efferentes de l'ecorce cerebrale. La Cellule 48:7-70.

Green, C.J. (1975) Neuroleptanalgesic drug combinations in the anaesthetic management of small laboratory animals. Lab. Anim. 9:161-178.

Gurdjian, E.S. (1927) The diencephalon of the albino rat. Studies on the brain of the rat. J. Comp. Neurol. 43:1-114.

Hellweg, F.C., R. Koch, and M. Vollrath (1977) Representation of the cochlea in the neocortex of guinea pigs. Exp. Brain Res. 29:467-474.

Hess, A. (1955) The nuclear topography and architectonics of the thalamus of the guinea pig. J. Comp. Neurol. 103:385-419.

Hose, B., G. Langner, and H. Scheich (1987) Topographic representation of periodicities in the forebrain of the Minah bird: One map for pitch and rhythm? Brain Res. 422:367-373.

Imig, T.J., and A. Morel (1983) Organization of the thalamocortical auditory system in the cat. Annu. Rev. Neurosci. 6:95-120.

Imig, T.J., and A. Morel (1984) Topographic and cytoarchitectonic organization of thalamic neurons related to their targets in low-, middle-, and high-frequency representations in cat auditory cortex. J. Comp. Neurol. 227:511-539.

Irvine, D.R.F. (1986) The Auditory Brainstem. A Review of the Structure and Function of Auditory Brainstem Processing Mechanisms. Berlin Springer Verlag.

Kayser, D., and J.P. Legouix (1963) Projections tonotopiques sur le cortex auditif du cobaye. C.R. Biol. 157:2161-2164.

Langner, G., and C. Schreiner (1987) Topology of functional parameters in the inferior colliculus of the cat. In N. Elsner and O.D. Creutzfeldt (eds): New Frontiers in Brain Research. Proceedings of the 15th Göttingen Neurobiology Conference. Stuttgart: Georg Thieme Verlag.

LeDoux, J.E., D.A. Ruggiero, and D.J. Reis (1985) Projections to the subcortical forebrain from anatomically defined regions of the medial geniculate body in the rat. J. Comp. Neurol. 242:182-213.

LeDoux, J.E., D.A. Ruggiero, R. Forest, R. Stornetta, and D.J. Reis (1987) Topographic organization of convergent projections to the thalamus from the inferior colliculus and spinal cord in the rat. J. Comp. Neurol. 264:123-146.

Mesulam, M.-M. (1982) Principles of horseradish peroxidase neurochemistry and their applications for tracing neural pathways--axonal transport, enzyme histochemistry and light microscopic analysis. In M.-M. Mesulam (ed): Tracing Neural Connections With Horseradish Peroxidase. Stadt: J. Wiley and Sons, pp. 1-152.

Mesulam, M.-M., and D.N. Pandya (1973) The projections of the medial geniculate complex within the Sylvian fissure of the Rhesus monkey. Brain Res. 60:315-333.

Middlebrooks, J.C., and J.M. Zook (1983) Intrinsic organization of the cat's medial geniculate body identified by projections to binaural responsespecific bands in the primary auditory cortex. J. Neurosci. 3:203-334

Mitani, A., and M. Shimokouchi (1985) Neuronal connections in the primary auditory cortex: An electrophysiological study in the cat. J. Comp. Neurol. 235:417-429

Morel, A., and T.J. Imig(1987) Thalamic projections to fields A, AI, P and VP in the cat auditory cortex. J. Comp. Neurol. 265:119-144.
Morest, D.K. (1964) The neuronal architecture of the medial geniculate body of the cat. J. Anat. 99:611-634.

Mori, J., N. Hori, and N. Katsuda (1980) A new method for application of horseradish peroxidase into a restricted area of the brain. Brain Res. Bull. 6:19-22.

Niimi, K., and H. Matsuoka (1979) Thalamocortical organization of the auditory system in the cat studied by retrograde axonal transport of horseradish peroxidase. Adv. Anat. Embryol. Cell Biol. 57:1-56.

Oliver, D.L., and W.C. Hall (1978a) The medial geniculate body of the tree shrew, Tupaia glis. I. Cytoarchitecture and midbrain connections. J. Comp. Neurol. 182:423-458.

Oliver, D.L., and W.C. Hall (1978b) The medial geniculate body of the tree shrew, Tupaia glis. II. Connections with the neocortex. J. Comp. Neurol. 182:459-494.

Oswaldo-Cruz, E., and C.E. Rocha-Miranda (1968) The Brain of the Opossum (Didelphis marsupialis). Rio de Janeiro: Instituto de Biofisica, Universidade Federal do Rio de Janeiro.

Patterson, H.A. (1977) An Anterograde Degeneration and Retrograde Axonal Transport Study of the Cortical Projections of the Rat Medial Geniculate Body. Boston University Graduate School, Ph.D.

Reale, R.A., and T.J. Imig (1980) Tonotopic organization in auditory cortex of the cat. J. Comp. Neurol. 192:265-291.

Redies, H., and S. Brandner (1987) New fields in the auditory cortex of the guinea pig. In N. Elsner and O.D. Creutzfeldt (eds): New Frontiers in Brain Research. Proceedings of the 15th Göttingen Neurobiology Conference. Stuttgart: Georg Thieme Verlag.

Redies, H., and O.D. Creutzfeldt (1987) The auditory thalamocortical system of the guinea pig. Neuroscience [Suppl.] 22:2153P (Abstr.).

Redies, H., U. Sieben, and O.D. Creutzfeldt (1988) Functional subdivisions in the auditory cortex of the guinea pig. J. Comp. Neurol. 280.000-000.

Robertson, D., and D.R.F. Irvine (1987) Functional plasticity of frequency maps in the guinea pig primary auditory cortex. Symposium: Auditory pathway--Structure and function. Prague, August 23-26 (Abstr.).

Scheel, M. (1987) Die Topographie der thalamocorticalen Verbindungen der Hörbahn bei der Ratte. Dissertation Universität Tübingen.

Schreiner, C., and M.S. Cynader (1984) Basic functional organization of second auditory field (AII) of the cat. J. Neurophysiol. 51:1284-1305.

Schreiner, C., and G. Langner (1988) Periodicity coding in the inferior colliculus of the cat. II. Topographical organization. J. Neurophysiol. 60:1823-1846.

Völker, H., and W. Graef (1965) Zytoarchitektonik der Zwischen-MittelhirnRegion beim Meerschweinchen (Cavia porcellus L.). Arch. Exp. Veterinärmed. 19:1121-1137.

Völker, H., and W. Graef (1966) Kerngebiete der Zwischen-MittelhirnRegion beim Meerschweinchen (Cavia porcellus L.). J. Hirnforsch. 9:285312 .

Winer, J.A. (1985) The medial geniculate body of the cat. Adv. Anat. Embryol. Cell Biol. 86:1-98.

Winer, JA, and D.T Larue (1987) Patterns of reciprocity in auditory thalamocortical and corticothalamic connections: Study with horseradish peroxidase and autoradiographic methods in the rat medial geniculate body. J. Comp. Neurol. 257:282-315.

Winer, J.A., I.T. Diamond, and D. Raczkowski (1977) Subdivisions of the auditory cortex of the cat: The retrograde transport of horseradish peroxidase to the medial geniculate body and posterior thalamic nuclei. J. Comp. Neurol. 176:387-418. 